\title{
Derivation of coarse-grained force fields for buckling-induced topological defects of liquid crystals
}

\author{
Michiaki Sakamoto (1) and Itsuo Hanasaki ${ }^{*} *$ \\ Institute of Engineering, Tokyo University of Agriculture and Technology, Naka-cho 2-24-16, Koganei, Tokyo 184-8588, Japan
}

(Received 24 December 2020; revised 12 July 2021; accepted 22 July 2021; published 31 August 2021)

\begin{abstract}
Microscopic details of buckling-induced topological defects are required for molecular design of smectic liquid crystals to control buckling instability of the layers. In this study, we present a multiobjective optimization method to derive the coarse-grained (CG) force fields with sufficiently precise buckling characteristics including the molecular details for molecular dynamics (MD) simulations. We perform CGMD simulations of buckling deformation at sample points in the CG force field parameter space, from which the response surfaces of objective functions such as the scalar orientational order parameters, critical angles of layer collapse, and radial distribution functions are estimated. Since not all objective functions can be optimized simultaneously, we use a genetic algorithm to calculate the Pareto set of optimal solutions. We select the models with different molecular head-tail symmetries to study buckling deformation. The extracted CG model successfully reproduces the buckling deformation in terms of the collapse of smectic layers through the generation of dislocations with dipole disclinations. We also find that the molecular symmetry is a dominant factor to control the class of the buckling-induced dislocations.
\end{abstract}

DOI: 10.1103/PhysRevE.104.024704

\section{INTRODUCTION}

Liquid crystals (LC) have both of the fluidity and the ordered structures, and they show various phases. In particular, the lamellar structures of the smectic phase with a twodimensional order [1] are observed not only in thermotropic LCs but also in ionic LCs, copolymers, and lipid bilayers. Therefore, the smectic phase has drawn widespread interest in the fields of nanopatterning manufacturing [2,3] and flexible electronic devices of displays, photonics, and semiconductors [4-6]. When the curvature of the layer reaches a critical value due to compressive strain, the lamellar structure collapses with the generation of topological defects. These defects are classified into two types: disclinations as the rotational symmetry breaking defects and dislocations as the translational symmetry breaking defects. The smectic-isotropic transition occurs in the core of these defects, which is considered to be a second-order phase transition of the Kosterlitz-Thouless type [7,8]. However, recent advances in transmission electron microscopy and fluorescence microscopy have made it possible to observe the molecular state of these defects, and the LC director configuration around singular cores has been clarified experimentally. Reference [9] shows that the lamellar structure of smectic LCs confined in prolate spheroids collapses to form a misorientation domain consisting of edge dislocations with disclination

\footnotetext{
*hanasaki@cc.tuat.ac.jp
}

Published by the American Physical Society under the terms of the Creative Commons Attribution 4.0 International license. Further distribution of this work must maintain attribution to the author(s) and the published article's title, journal citation, and DOI. pairs. They have found that the curvature of the layer increases as the aspect ratio of LC droplets increases. When the critical angle $\gamma$ exceeds $\gamma=50^{\circ}-70^{\circ}$, the apex of the undulated layer collapses and dislocations with disclination pairs are generated. Similar dislocations with disclination pairs have been observed in Grandjean-Cano wedges [10] filled with a cholesteric LC using a fluorescence microscope [11]. Therefore, elucidating the mechanism of these phenomena and establishing a molecular design method for stabilizing the smectic layer are challenging issues for electronics and fabrication.

The molecular dynamics (MD) simulation allows us to investigate the phase states and thermodynamic quantities by taking the ensemble of calculated molecular trajectories, and hence appropriate for investigating the microscopic details of lamellar deformation. The director orientation of nematic LCs confined in a micrometer-scale cylinder has been studied with the united-atom MD, and it has been demonstrated that topological defects were formed around the inner diameter of the cylinder [12]. However, microsecond timescale sampling is necessary to analyze the nature of defects, since the topological defects generation is a relatively slow stochastic process. Coarse-grained (CG) MD has been useful to overcome this issue [13,14]. Reference [15] conducts the CGMD simulation and visualizes the topological defects of nematic LCs with Gay-Berne force fields. The shear-induced buckling instabilities of smectic LCs have been studied with the tetramer structure A-A-B-B consisting of CGMD particles [16]. These simulations provide the microscopic descriptions of the LC topological defects and the instabilities. However, since these force fields are not based on actual LC molecules, they cannot be analyzed in relation to the atomistic details. On the other hand, the Iterative Boltzmann Inversion (IBI) method [14] is a systematic approach to derive CG force fields from the 
all-atom models. The CG force fields of azobenzene LCs were derived from radial distribution function (RDF) of the supercooled isotropic state near the smectic-isotropic phase transition point using IBI method [17]. However, if the model is extracted from the RDF in the supercooled isotropic state, then it is not suitable to deal with large-strain deformations. Subtle thermal fluctuations can disorder the lamellar structure because of insufficient fitting to the smectic characteristics. Furthermore, the critical stress to generate the dislocations of LCs is orders of magnitude smaller than those to generate metal dislocations. The critical stress $\sigma_{\mathrm{c}}$ of LCs to generate dislocations is described as $\sigma_{\mathrm{c}}=\sqrt{K B} / d$, where $B$ is the compressibility modulus, $K$ is the Frank modulus which governs the curvature of elasticity, and $d$ is the thickness of sample [18-21]. It is estimated as $\sigma_{\mathrm{c}} \approx 10^{4} \mathrm{~Pa}[18,21]$ by taking $B \approx 10^{6} \mathrm{Nm}^{-2}, K \approx 10^{-10} \mathrm{~N}$, and $d \approx 10 \mu \mathrm{m}$, which is 6 orders of magnitude smaller than the critical stress $\sigma_{c} \approx 10^{10} \mathrm{~Pa}$ of metals [22]. Since small differences in the force fields can affect the buckling instability of the smectic layers, it is difficult to reproduce the buckling instabilities of the smectic LCs with the force field parameters extracted only from the structural properties in the undeformed state by the IBI method. The parameters need to be optimized using features that characterize the buckling-induced collapse of layers.

One approach for calibrating the force fields is to use a multiobjective optimization [23,24]. Genetic algorithm (GA) $[25,26]$ is an effective method because of the multipoint search and easy parallelization. Not a single but a set of solutions are obtained by sampling of the Pareto front [27] derived with GA. However, the calculations must be performed typically for more than $10^{5}$ sampling points in the calibration parameter space to derive the accurate Pareto set of optimal solutions. It is difficult to perform MD calculations for each of the sampling points due to time constraints. The response surface method (RSM) with Gaussian process (GP) is an efficient approach to search the optimal points of the multiobjective functions. Here, the response surface is defined as a surface to approximate the high dimensional manifold of the multiobjective functions on the decision variable space. The GP can estimate the rugged landscapes of the response surface with high accuracy. In Refs. [28-30], force fields of liquid argon and water have been modeled using the RSM with GP. Reference [31] employed the multiobjective optimization method based on RSM and GP to find the Pareto set of optimal solutions suitable for the target properties from a computational data set of piezoelectric compounds. Reference [32] used the multiobjective optimization of force field parameters for two-dimensional layered materials $2 \mathrm{H}-\mathrm{MoSe}_{2}$ using GA to reproduce several structural, thermodynamic and vibrational properties.

However, when the multiobjective optimization method is applied to the parametrization of the CG force field suitable for the buckling instability of smectic LC, there are several issues to be solved. Since the subtle difference of force field can alter the characteristics of LC model particularly when topological defects emerge, the decision variables and their search space need to be carefully examined, taking into account the molecular symmetry. Furthermore, it is not sufficient to reproduce the buckling deformation in terms of the collapse of smectic layers but the dominant factors to control the characteristics of buckling instability, such as plastic deformation and the type of buckling-induced defects, have to be consistent with the existing knowledge. In addition, it is also necessary to examine that the simulation results with the Pareto front solution satisfy the established topology theory of LC defects [10,33-35]. To the best of our knowledge, there has been no attempt to validate and classify the topological defects generated with the different models of the CGMD force fields from the topology perspective.

In this article, we present the multiobjective optimization method based on the Pareto set to derive the CG force field of LCs with sufficiently precise buckling characteristics including molecular details for MD. We assume the critical angle of the layer collapse and the orientational order parameter as the quantities of interest (QoI) to control the buckling instabilities of LCs. We also assume the correction factor of the intermolecular force field that represents the molecular symmetry as decision variables. We divide the search space into subdomains with high and low molecular symmetries to find the optimum solution in each subdomain. Defining these objective functions and decision variables in the multiobjective optimization scheme, we successfully calibrate the tentative force fields derived by the IBI method. We show that the derived models reproduce experimental data on the collapse of the layer due to buckling deformation and the generation of edge dislocations with dipole disclination pairs $[9,11]$. We also show the derived models are consistent with the topological defects theory [10,33-35], and clarify the relation between the molecular symmetry and the buckling-induced topological defects.

\section{METHODOLOGY}

\section{A. United-atom model to be coarse-grained}

A reference $\mathrm{LC}$ model is required to develop the $\mathrm{CG}$ model that reproduces the phenomena in the above-mentioned experiments. We use 4'-octyl-4-biphenylcarbonitrile, i.e., so-called $8 \mathrm{CB}$, as a smectic LC molecule. The $8 \mathrm{CB}$ model described in the literature [36] was optimized to match the experimental values of the phase transition temperature, and it is not suitable for reproducing the bending deformation of the smectic layers. Therefore, we develop a reference $8 \mathrm{CB}$ model suitable for bending deformation using the general Amber force field (GAFF) of Amber18 [37]. The structure of the $8 \mathrm{CB}$ molecule used in the united-atom simulations is shown in Fig. 1(a). Since the GAFF force field is an all-atom model with explicit modeling of $\mathrm{H}$ atoms, the $\mathrm{H}$ atoms are merged to each bonded atom to define the force field of the united-atom model. We use the correction model of the dihedral angular potential based on gas-phase electron diffraction measurements [36,38]. We use Amber's bond charge correction model (AM1-BCC) $[39,40]$ as the charge model to be defined here.

MD simulations are performed with the GROMACS simulation package [41]. We consider a unit domain of a four-layered smectic lamellar system with a projected area of about $8.2 \times 8.2 \mathrm{~nm}^{2}$ in the $x y$ plane under the periodic boundary conditions as the initial setup. To reproduce the experimental double-layer smectic phase $\mathrm{SmA}_{\mathrm{d}}$, each layer 
(a)

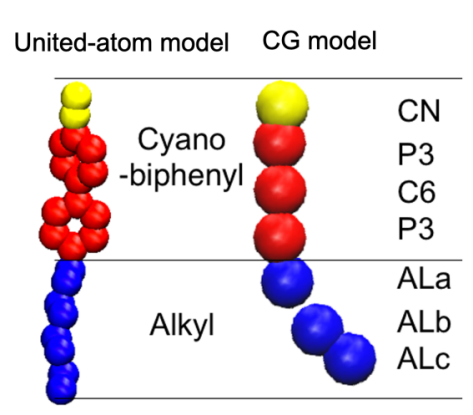

(b)
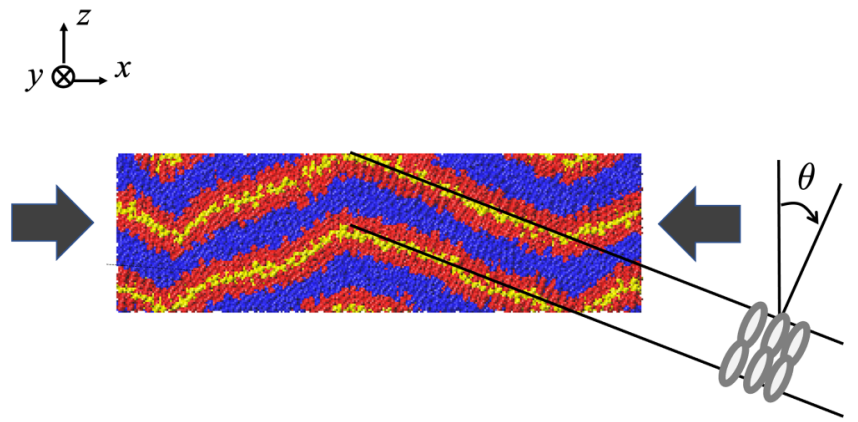

FIG. 1. The 8CB molecule and their smectic lamellar cell: (a) The structure of the united-atom model, and the corresponding structure of the CG model, and (b) example of the chevron texture during the buckling deformation of the smectic lamellar cell with the definition of the coordinate and the layer rotation angle $\theta$.

consists of two sublayers of molecules aligned in the opposite directions with each other along the $z$ axis of the simulation box. The simulation is carried out for $20 \mathrm{~ns}$ using a time step of 2 fs under the NPT ensemble at a temperature of $300 \mathrm{~K}$ and a pressure of $10^{5} \mathrm{~Pa}$. Electrostatic interactions are computed using the particle mesh Ewald method, and van der Waals interactions are applied with a cutoff of $1.5 \mathrm{~nm}$ and a potential shift with Verlet cutoff scheme. The Berendsen weak coupling algorithm [42] is used to control both temperature and pressure. The coupling constant of the thermostat is $1 \mathrm{fs}$ which provides the diffusion coefficients consistent with the experimental values measured by the single molecule tracking [43-45]. The time constant of the barostat is $1 \mathrm{~ns}$ and the isothermal compressibility to $10^{-5} \mathrm{bar}^{-1}$ so that the relaxation time constant $\tau$ of the LC molecular deformation is consistent with that of the LC molecular fluctuation obtained by quasielastic gamma spectroscopy [46]. The calculated dipole moment is $\mu=5.0$, which is within the range, but close to the lower limit of the reported value $\mu=4.7-8.0$ [47-49] based on the charge distribution in the optimal atomic configuration of the molecule with Hartree-Fock calculations. Therefore, we modify the cyano group charge to achieve the dipole moment $\mu=6.2$. The comparison of dipole moments $\mu$, diffusion coefficients $D$, scalar orientational order parameters $S$, and deformation relaxation time constants $\tau$ between our simulation and the reference values of the recent computational and experimental work in the literature are shown in Table I. Here the scalar order parameter $S$ is defined by Eq. (A1), where the vector $\boldsymbol{d}$ is the molecular long axis between the particles at both ends of biphenyl group. The calculated physical quanti-

TABLE I. Comparison of dipole moments $\mu$, scalar orientational order parameters $S$ [cf. Eq. (A1)], diffusion coefficients $D$, and deformation relaxation time constants $\tau$ between this study and the recent computational and experimental work in the literature.

\begin{tabular}{|c|c|c|c|}
\hline & & This work & References \\
\hline$\mu$ & & 6.2 & $4.7-8.0$ [47-49] \\
\hline$S$ & & 0.65 & $0.6-0.7[50,51]$ \\
\hline$D$ & $\left(\times 10^{-12} \mathrm{~m}^{2} / \mathrm{s}\right)$ & 0.86 & $2-30[43,45,50]$ \\
\hline$\tau$ & (ns) & 20 & $10-100[52,53]$ \\
\hline
\end{tabular}

ties reproduce the reference values well and can be used as the basis for the CG model.

\section{B. Mapping to the CG model and the effective molecular interactions}

The mapping scheme for $8 \mathrm{CB}$ molecules to $\mathrm{CG}$ particles is shown in Fig. 1. The CG model consists of cyanobiphenyl and alkyl groups using four different types of particles. The head cyano group consists of one $\mathrm{CN}$ particle, the rigid biphenyl group consists of two P3 particles with one C6 particle, and the tail alkyl group consists of three AL particles. The effective molecular interaction of our model is described as

$$
\begin{aligned}
& W_{\mathrm{eff}}\left(b^{1}, \ldots, b^{N_{\mathrm{b}}}, \phi^{1}, \ldots, \phi^{N_{\mathrm{a}}}, r^{12}, \ldots, r^{N-1, N}\right) \\
& =\sum_{i=1}^{N_{b}} U_{\mathrm{bond}}\left(b^{i}\right)+\sum_{i=1}^{N_{a}} U_{\text {angle }}\left(\phi^{i}\right)+\sum_{i=1}^{N-1} \sum_{j=i+1}^{N} U_{\mathrm{LJ}}\left(r^{i j}\right),
\end{aligned}
$$

where $N_{\mathrm{b}}$ and $N_{\mathrm{a}}$ are the total number of bonds and bond angles, respectively, and $N$ is the number of beads in the system. The $b^{i}$ and $\phi^{i}$ represent the $i$ th bond length and the $i$ th bond angle, respectively, and $r^{i j}$ represents the distance between the $i$ th and $j$ th particles. The first and second terms on the right side are intramolecular potentials, where $U_{\text {bond }}$ is the bond-stretching potential and $U_{\text {angle }}$ is the angle-bending potential. Using the harmonic potential, each term is approximated by

$$
\begin{gathered}
U_{\text {bond }}\left(b^{i}\right)=\frac{1}{2} K_{\mathrm{b}}^{i}\left(b^{i}-b_{0}^{i}\right)^{2}, \\
U_{\text {angle }}\left(\phi^{i}\right)=\frac{1}{2} K_{\mathrm{a}}^{i}\left(\phi^{i}-\phi_{0}^{i}\right)^{2},
\end{gathered}
$$

where $K_{\mathrm{b}}^{i}, K_{\mathrm{a}}^{i}$ are force constants of bond-stretching and angle-bending potentials, respectively, and $b_{0}^{i}, \phi_{0}^{i}$ are their equilibrium positions. The intermolecular potential is represented by Lennard-Jones potential:

$$
U_{\mathrm{LJ}}\left(r^{i j}\right)=4 \epsilon^{i j}\left[\left(\frac{\sigma^{i j}}{r^{i j}}\right)^{12}-\left(\frac{\sigma^{i j}}{r^{i j}}\right)^{6}\right],
$$

where $\epsilon^{i j}$ and $\sigma^{i j}$ are the energy and size parameters, respectively. 
TABLE II. Parameters of bond-stretching and angle-bending interactions [cf. Eq. (2)] of the 8CB CG model. The parameters of types WP and NP are extracted from the range with and without the plateau region of the target potential, respectively.

\begin{tabular}{lccccc}
\hline \hline Bond & $\begin{array}{c}b_{0}^{i} \\
(\mathrm{~nm})\end{array}$ & $\begin{array}{c}K_{\mathrm{b}}^{i} \\
\left(\mathrm{~kJ} \mathrm{~mol}^{-1} \mathrm{~nm}^{-2}\right)\end{array}$ & Angle & $\begin{array}{c}\phi_{0}^{i} \\
(\mathrm{degree})\end{array}$ \\
\hline CN-P3 & 0.242 & Rigid & CN-P3-C6 & 188.7 & $K_{\mathrm{a}}^{i}$ \\
$\left(\mathrm{~kJ} \mathrm{~mol}^{-1} \mathrm{rad}^{-2}\right)$
\end{tabular}

\section{Initial CG force field}

We derive the initial $\mathrm{CG}$ force field parameters based on the mapping defined in Fig. 1 from the united-atom model by the IBI method. The IBI is performed using the VOTCA package [54]. The unit domain size is the same as the united-atom model. The simulation is performed in the NPT ensemble at a temperature of $300 \mathrm{~K}$ and a pressure of $10^{5} \mathrm{~Pa}$ using the stochastic dynamics thermostat with a coupling time coefficient of $1 \mathrm{fs}$. The pressure is controlled by the weak coupling algorithm with a compressibility of $10^{-5} \mathrm{bar}^{-1}$ and a coupling constant of $10 \mathrm{ps}$. We used a time step of $2 \mathrm{fs}$ and the simulation duration is $0.5 \mathrm{~ns}$. If the tentative intermolecular interaction is not sufficiently precise, then the nematic phase is not preserved in a time scale of $10 \mathrm{~ns}$. Therefore, the sampling period of the IBI method is set as short as $0.5 \mathrm{~ns}$, and it is assumed that the potential is improved at the multiobjective step by performing the MD calculation with a sampling period of $20 \mathrm{~ns}$, which is sufficient to equilibrate the nematic phase. The bonded interactions $U_{\text {bond }}, U_{\text {angle }}$ are derived by the Boltzmann inversion method to reproduce the distribution $P$ of bond lengths $b^{i}$ and bond angles $\phi^{i}$ :

$$
U(q)=-k_{\mathrm{B}} T \ln P(q),
$$

where $U$ is either $U_{\text {bond }}$ or $U_{\text {angle }}, q$ is either $b^{i}$ or $\phi^{i}, k_{\mathrm{B}}$ is Boltzmann constant, and $T$ is the temperature of the system. The obtained $U_{\text {bond }}, U_{\text {angle }}$ are approximated by the harmonic potentials of Eq. (2). The extracted intramolecular force field parameters $K_{\mathrm{b}}^{i}, K_{\mathrm{a}}^{i}, b_{0}^{i}$, and $\phi_{0}^{i}$ are shown in Table II. A plateau appears at the angle bending potential $U_{\text {angle }}$ in the alkyl groups of $8 \mathrm{CB}$ because of the high flexibility. Different parameter sets are extracted depending on whether this plateau is included or not. We denote the angle bending potential parameters with the plateau by type WP and those without the plateau by type NP.

The intermolecular interactions $U_{\mathrm{LJ}}$ are also extracted with the Boltzmann inversion method, and then fit with the LJ potentials of Eq. (3). The approximation is applied in the range from the intermolecular distance $r=0.3 \mathrm{~nm}$ to the first maximum peak. Then, the potential $U_{\mathrm{LJ}}$ is updated till convergence by IBI method as:

$$
\begin{aligned}
& U^{(n+1)}\left(r^{i j}\right)=U^{(n)}\left(r^{i j}\right)+\Delta U^{(n)}\left(r^{i j}\right), \\
& \Delta U^{(n)}\left(r^{i j}\right)=k_{\mathrm{B}} T \ln \frac{g_{0}^{(n)}\left(r^{i j}\right)}{g_{0}^{\mathrm{UA}}\left(r^{i j}\right)},
\end{aligned}
$$

where $g_{0}^{(n)}\left(r^{i j}\right)$ is the RDF of the $n$th entry of the CG model and $g_{0}^{\mathrm{UA}}\left(r^{i j}\right)$ is the RDF of the target united-atom model. The tabulated potential after the convergence produces the target distribution. Since intermolecular force fields are strongly correlated with each other, the $\mathrm{CN}-\mathrm{CN}$ interaction is fixed and other intermolecular potentials are extracted by the IBI method. The extracted force fields are then approximated by the LJ potential again for smoothing. The derived parameters are shown in Table III. The flexible alkyl group interaction, i.e., type WP in Table II is employed for the intramolecular interaction. The derived force field does not reproduce the properties of buckling-induced collapse of the smectic system or their structural properties, as described in the following paragraphs. Thus, this is considered as the initial force field that needs to be calibrated by the multiobjective optimization. Hereafter, we refer to this force field as model 1.

We evaluate physical quantities such as the diffusion coefficient $D$, the scalar orientational order parameter $S$, and RDF $\mathrm{g}_{0}(r)$ for model 1 . The production simulation is performed for $20 \mathrm{~ns}$ using a time step of $10 \mathrm{fs}$ by NPT ensemble at a temperature of $300 \mathrm{~K}$ and a pressure of $10^{5} \mathrm{~Pa}$. The coupling constant of the thermostat is $1 \mathrm{fs}$, while the barostat has a coupling constant of $1 \mathrm{~ns}$ with compressibility of $10^{-5} \mathrm{bar}^{-1}$. In addition, the simulation of bending deformation is performed to evaluate the critical angle. We configure a bilayered smectic cell consisting of two interdigitated sublayers with a projected area of about $31.4 \times 7.9 \mathrm{~nm}^{2}$ in the $x y$ plane under the periodic boundary conditions. Each layer consists of two sublayers of molecules aligned in the opposite direction to form the smectic $\mathrm{SmA}_{\mathrm{d}}$ phase, and the thickness of the layer is $3.8 \mathrm{~nm}$. In case the bending simulation is performed from the

TABLE III. Parameters of LJ potentials [cf. Eq. (3)] for the intermolecular interactions of model 1 . The parameters of type WP in Table II are adopted as the intramolecular interactions [cf. Eq. (2)].

\begin{tabular}{lccccc}
\hline \hline CG-CG & $\begin{array}{c}\sigma \\
(\mathrm{nm})\end{array}$ & $\begin{array}{c}\epsilon \\
\left(\mathrm{kJ} \mathrm{mol}^{-1}\right)\end{array}$ & CG-CG & $\begin{array}{c}\sigma \\
(\mathrm{nm})\end{array}$ & $\begin{array}{c}\epsilon \\
\left(\mathrm{kJ} \mathrm{mol}^{-1}\right)\end{array}$ \\
\hline CN-CN & 0.314 & 3.993 & CN-P3 & 0.360 & 2.473 \\
P3-P3 & 0.468 & 0.946 & CN-C6 & 0.374 & 1.824 \\
C6-C6 & 0.487 & 0.922 & CN-AL & 0.350 & 2.870 \\
AL-AL & 0.377 & 2.573 & P3-C6 & 0.469 & 0.469 \\
& & & P3-AL & 0.433 & 0.618 \\
& & C6-AL & 0.373 & 0.921 \\
\hline \hline
\end{tabular}


TABLE IV. Parameters of LJ potentials [cf. Eq. (3)] for the intermolecular interactions to prepare the sinusoidal shape as the initial setup for the bending simulation.

\begin{tabular}{cccccc}
\hline \hline CG-CG & $\begin{array}{c}\sigma \\
(\mathrm{nm})\end{array}$ & $\begin{array}{c}\epsilon \\
\left(\mathrm{kJ} \mathrm{mol}^{-1}\right)\end{array}$ & CG-CG & $\begin{array}{c}\sigma \\
(\mathrm{nm})\end{array}$ & $\begin{array}{c}\epsilon \\
\left(\mathrm{kJ} \mathrm{mol}^{-1}\right)\end{array}$ \\
\hline CN-CN & 0.345 & 0.647 & CN-P3 & 0.409 & 2.190 \\
P3-P3 & 0.441 & 0.735 & CN-C6 & 0.408 & 1.396 \\
C6-C6 & 0.454 & 0.562 & CN-AL & 0.388 & 0.690 \\
AL-AL & 0.416 & 0.742 & P3-C6 & 0.448 & 0.836 \\
& & & P3-AL & 0.489 & 0.515 \\
& & & C6-AL & 0.519 & 0.386 \\
\hline \hline
\end{tabular}

flat shape using model 1 , the directors tend to tilt from the normal direction to the layer and disorder the $\mathrm{SmA}_{\mathrm{d}}$ state in the sudden change of the flat shape during the initial compression. Therefore, we prepare the slightly undulated cell of the $\mathrm{SmA}_{d}$ state with the layer angles of about $10^{\circ}$ as the initial setup. We compress the flat smectic cell in the $x$ direction and pull it in the $z$ direction at the same time with the different force field based on that described in Ref. [55] to deform the flat layers into a sinusoidal shape. The LJ potential parameters that we use here are shown in Table IV, and the intramolecular parameters are the same as those of model 1. Its structural properties are not consistent with those of the united-atom model and the interactions between cyanobiphenyl particles are stronger than the other interactions including the terminal groups such as $\mathrm{CN}$ and $\mathrm{AL}$ particles, which does not accurately describe the mechanism of smectic phase formation of $8 \mathrm{CB}$ molecules by the polar terminal substituents [56]. However, we use the force field to generate the sinusoidal shape, since $S m A_{d}$ phase is not disordered at the buckling. After the preparation of the initial configuration of the system, we switch the force field to model 1 . We equilibrate the slightly deformed system using model 1 with the fixed boundary condition in the $x$ and $y$ directions. Then, the system is compressed in the $x$ direction with the strain rate of $d \varepsilon / d t=0.78 \mu \mathrm{s}^{-1}$ using model 1 to evaluate the critical angle. The deformation is carried out over a period of $800 \mathrm{~ns}$ corresponding to the strain of 0.624 using a time step of $10 \mathrm{fs}$ until the layers collapse to estimate the critical angle $\gamma$.

Table X shows the comparison of the physical properties of Model 1, the united-atom model, and experimental data by the scalar orientational order parameter $S$, the layer critical angle $\gamma$, the difference of RDF peak heights of C6-C6 particles from the target united-atom model, and the difference of the density distribution $G_{z}(z)$ of $\mathrm{CN}$ particles at the position $z$ along the layer normal. The RDF is defined as

$$
g_{0}(r)=\frac{1}{4 \pi r^{2} \rho_{0}}\left\langle\delta\left(r-r^{i j}\right)\right\rangle,
$$

where $\rho_{0}$ the average density of particles and $r^{i j}$ is the distance between the $i$ th and $j$ th particles. The density distribution $G_{z}(z)$ of $\mathrm{CN}$ particles at the position $z$ along the layer normal is defined as

$$
G_{z}(z)=\frac{1}{\rho_{0}}\left\langle\delta\left(z-z^{i}\right)\right\rangle,
$$

where $z^{i}$ is the $z$ position of the $i$ th particle. The differences in RDF peak heights are defined as

$$
\triangle g_{0}(k)=\frac{g_{0}^{\mathrm{CG}}(k)}{g_{0}^{\mathrm{UA}}(k)}-1(k=1,2),
$$

where $g_{0}^{l}(k)(l=\mathrm{CG}$, UA) are the $k$ th peak heights of the RDFs of $C G$ and the united-atom models, respectively. The difference $\triangle G_{z}(z)$ is defined in the same way. The scalar orientational order parameter $S$ is defined as Eq. (A1), where the vector $\boldsymbol{d}$ is the molecular long axis between P3-P3 particles.

The critical angle $\gamma$ is estimated from the director angle in the layer outside the misorientation domain at the time of layer collapse. As shown in Table X, the scalar orientational order parameter $S=0.758$ of model 1 is larger than the experimental value $S=0.6-0.7$. There are also significant deviations from the target $\mathrm{RDF} g_{0}(r)$ and the density distribution $G_{z}(z)$ along the vertical direction of layers. The critical angle $\gamma=60^{\circ}$ of model 1 is comparable to the experimental value.

\section{Multiobjective optimization method and the derived CG force fields for the actual use}

\section{Decision variable}

To calibrate the intermolecular force field, correction factors $p_{u(i) v(j)}$ are introduced as follows:

$$
W_{\text {inter }}\left(r^{i j}\right)=\sum_{i=1}^{N-1} \sum_{j=i+1}^{N} p_{u(i) v(j)} U_{\mathrm{LJ}}\left(r^{i j}\right),
$$

where $W_{\text {inter }}\left(r^{i j}\right)$ is the calibrated inter-particle interactions, and $u(i)$ and $v(j)$ are the types of the $i$ th and $j$ th CG particles, respectively. We determine the correction factor of the $\mathrm{CN}-\mathrm{CN}$ interaction $p_{1}=p_{\mathrm{CN}, \mathrm{CN}}$ and that of the AL-AL interaction $p_{2}=p_{\mathrm{AL}, \mathrm{AL}}$ as the decision variables $\boldsymbol{p}=\left(p_{1}, p_{2}\right)$ since the interactions of terminal substituents determine the molecular symmetry and have dominant effects on the formation of the smectic phase. The interactions between P3-P3 and C6-C6 are not modified, and the others are modified by the correction factor $p_{u(i) v(j)}=\sqrt{p_{u(i) u(i)} p_{v(j) v(j)}}$. The range of the search space is defined to be $0.2<p_{k}<1.0(k=1,2)$. We divide the search space into subdomains $p_{1}<p_{2}$ and $p_{1}>p_{2}$ to find the optimal solution taking into account the molecular head-tail symmetry. The optimal force fields for $p_{1}<p_{2}$ have more head-tail symmetry than those for $p_{1}>p_{2}$, since the energy parameter $\epsilon_{\mathrm{CN}, \mathrm{CN}}$ is larger than $\epsilon_{\mathrm{AL}, \mathrm{AL}}$ in the initial intermolecular force field in Table III.

\section{Objective functions}

The following are the QoIs to be assimilated with the experimental and simulated data: (1) the scalar orientational order parameter $S$ in the range of $0.60<S<0.70$, (2) the RDF of the reference system, and (3) the critical angle $\gamma$ of the layer collapse in the range of $45^{\circ}<\gamma<65^{\circ}$.

The loss function $L_{1}(\boldsymbol{p})$ of the order parameter $S$ is defined as

$$
\begin{aligned}
L_{1}(\boldsymbol{p}) & =h[\hat{S}(\boldsymbol{p})], \\
\hat{S}(\boldsymbol{p}) & =\frac{S(\boldsymbol{p})-S^{\min }}{S^{\max }-S^{\min }},
\end{aligned}
$$


where $h(x)$ is the penalty function, $\hat{S}$ is the normalized function of $S, S^{\mathrm{min}}$ is the minimum $S^{\min }=0.60$, and $S^{\max }$ is the maximum $S^{\max }=0.70$. The penalty function $h(x)$ is a double sigmoid curve defined as

$$
h(x)=\frac{1}{1+\exp (-\alpha x)}+\frac{1}{1+\exp [-\alpha(1-x)]} .
$$

The inflection points of the curves are located at $x=0$ and 1 . The curve approaches asymptotically to $h(x) \rightarrow 0$ in the range of $0<x<1$, and $h(x) \rightarrow 1$ otherwise. $\alpha$ is a hyperparameter for the ascending and descending slopes around the inflection points. $\alpha$ is set to $\alpha=10$ so as to be $L_{1}>0.9$ in the range of $S<0.577$ or $S>0.723$, and to be $L_{1}<0.1$ in the range of $0.623<S<0.777$. The specific method to determine the hyperparameter value will be discussed later.

The RDF loss function $L_{2}(\boldsymbol{p})$ is defined as

$$
L_{2}(\boldsymbol{p})=a_{2} \sum_{i \in \mathrm{C} 6, \mathrm{P} 3} \sum_{k=1}^{2}\left[\frac{g_{0 i}^{\mathrm{CG}}(k, \boldsymbol{p})}{g_{0 i}^{\mathrm{UA}}(k)}-1\right]^{2} .
$$

$g_{0 i}^{\mathrm{CG}}(1, \boldsymbol{p})$ and $g_{0 i}^{\mathrm{CG}}(2, \boldsymbol{p})$ are the first and second peak heights of the RDF of the CG particles $(i \in \mathrm{C} 6, \mathrm{P} 3)$, respectively. $g_{0 i}^{\mathrm{UA}}(1), g_{0 i}^{\mathrm{UA}}(2)$ are those of the united-atom model. $a_{2}$ is a normalization factor.

The loss function $L_{3}(\boldsymbol{p})$ of the critical angle $\gamma$ is defined as

$$
\begin{aligned}
& L_{3}(\boldsymbol{p})=h[\hat{\gamma}(\boldsymbol{p})], \\
& \hat{\gamma}(\boldsymbol{p})=\frac{\gamma(\boldsymbol{p})-\gamma^{\min }}{\gamma^{\max }-\gamma^{\min }},
\end{aligned}
$$

where the explicit form of $h(x)$ is Eq. (11), $\hat{\gamma}$ is the normalized function of $\gamma, \gamma^{\min }$ is the minimum $\gamma^{\min }=45^{\circ}$, and $\gamma^{\max }$ is the maximum $\gamma^{\max }=65^{\circ}$. The value of $\alpha$ is the same as that of $L_{1}$ so as to be $L_{3}<0.1$ in the range of $49.5^{\circ}<\gamma<60.5^{\circ}$ and $L_{3}>0.9$ in the range of $\gamma<40.5^{\circ}$ or $\gamma>69.5^{\circ}$.

We define the parameter constraint function $C_{1}(\boldsymbol{p})$ as

$$
\begin{aligned}
C_{1}(\boldsymbol{p}) & =C_{1}\left(p_{1}, p_{2}\right)=h\left(\hat{p}_{1}\right)+h\left(\hat{p}_{2}\right), \\
\hat{p}_{j} & =\frac{p_{j}-p_{j}^{\min }}{p_{j}^{\max }-p_{j}^{\min }}(j=1,2),
\end{aligned}
$$

where $p_{j}^{\min }$ and $p_{j}^{\max }$ are the minimum and maximum in the search space defined as $p_{j}^{\min }=0.2$ and $p_{j}^{\max }=1.0$. We also define the parameter constraint function $C_{2}(\boldsymbol{p})$ as

$$
C_{2}(\boldsymbol{p})=C_{2}\left(p_{1}, p_{2}\right)=h\left(\hat{p}_{2}-\hat{p_{1}}\right),
$$

which is equivalent to the constraint condition $p_{1}<p_{2}$. Similarly, the parameter constraint function $C_{3}(\boldsymbol{p})$ is defined as

$$
C_{3}(\boldsymbol{p})=C_{3}\left(p_{1}, p_{2}\right)=h\left(\hat{p}_{1}-\hat{p_{2}}\right),
$$

which is equivalent to the constraint condition $p_{1}>p_{2}$.

The objective functions $f_{j}(\boldsymbol{p})(j=1,2,3)$ are defined as

$$
f_{j}(\boldsymbol{p})=L_{1}(\boldsymbol{p})+\beta C_{1}(\boldsymbol{p})+\beta C_{k}(\boldsymbol{p}),
$$

where $k=2$ for $p_{1}<p_{2}$ and $k=3$ for $p_{1}>p_{2} . j=1,2,3$ corresponds to the cases of orientational order parameter $S$, RDF $g_{0}$, and critical angle $\gamma$, respectively. The first term of the right-hand side is the loss function, and the second and the third terms are the constraints on the decision variables. The hyperparameter $\beta$ is set to $\beta=10^{6}$. The specific method to determine the hyperparameter value will be discussed later.

\section{Multiobjective optimization algorithm to extract the CG force fields}

The outline of the multiobjective optimization procedure for the force field calibration proposed in this work is the following.

(1) Generating the initial sampling points.

The LJ parameters are extracted by fitting the tabular intermolecular potential estimated with the IBI method. Decision variables $\boldsymbol{p}_{i}\left(i=1, \ldots, N_{\mathrm{s}}\right)$, i.e., the correction factors of the LJ parameters are randomly generated in the search space, where $N_{\mathrm{s}}$ is the number of sampling points of MD calculations.

(2) Modeling the response surface.

The values of loss functions $y_{i}^{1}, y_{i}^{2}$ and $y_{i}^{3}\left(i=1, \ldots, N_{\mathrm{s}}\right)$ for each objective function are obtained by performing MD calculations on $\boldsymbol{p}_{i}$. Each response surface $f_{j}(j=1,2,3)$ is calculated from the training data $\left\{\boldsymbol{p}_{i}, y_{i}^{1}, y_{i}^{2}, y_{i}^{3}\right\}$ using GP.

(3) Exploring the Pareto optimal solutions set.

The optimal value of each response surface is searched using GA. The calculation is performed 10 times with a population size of 200 and the number of generations of 200 to derive the Pareto set $\Omega_{\text {Pareto }}$.

(4) Checking the convergence.

The convergence is judged by $\left|\boldsymbol{p}_{i \text {,curr }}^{\mathrm{opt}}-\boldsymbol{p}_{i, \mathrm{prev}}^{\mathrm{opt}}\right|<\delta$ for each response surface, where $\delta$ is set to $\delta=0.03$. $\boldsymbol{p}_{i, \text { curr }}^{\text {opt }}$ and $\boldsymbol{p}_{i, \text { prev }}^{\text {opt }}$ are the optimal points of the response surfaces in the current and the previous processes, respectively. When the convergence criteria are satisfied, the iteration is terminated, and the process proceeds to Step 6.

(5) Exploring the next sampling points.

The point $\boldsymbol{p}_{i, \mathrm{Eurr}}^{\mathrm{EI}}$ that maximizes the expected improvement acquisition function (EI) of the Bayesian optimization [57] and several previously unselected points around it are added to the next sampling points. The optimal solution $\boldsymbol{p}_{i, \mathrm{curr}}^{\mathrm{opt}}$ for each response surface is also added to the sampling points. The process then returns to Step 2 to improve the accuracy of the response surface.

(6) Selecting the optimal solution.

The converged response surfaces are searched again to obtain the final Pareto optimal solution set $\left\{\boldsymbol{p} \mid \boldsymbol{p} \in \Omega_{\text {Pareto }}\right\}$. The solution suitable for the buckling simulation is selected from the final Pareto set. Their statistical distributions are also estimated.

\section{The Pareto optimal solutions set of the force fields and the selected CG models}

We calibrate the initial LJ parameters based on the multiobjective optimization algorithm mentioned above. We find the optimal solutions for each of $p_{1}<p_{2}$ and $p_{1}>p_{2}$, respectively. The hyperparameters are set to $\alpha=10$ and $\beta=$ $10^{6}$. The derived response surfaces for $p_{1}<p_{2}$ are shown in Figs. 2(a)-2(c). The optimal ranges of the objective functions $f_{1}, f_{2}$, and $f_{3}$ corresponding to order parameter, RDF, and critical angle are around $\left(p_{1}, p_{2}\right)=(0.6,0.7),(0.3,0.5)$, and $(0.7,0.85)$, respectively. Each objective function has a different optimal range and there are trade-offs between them. 
(a)

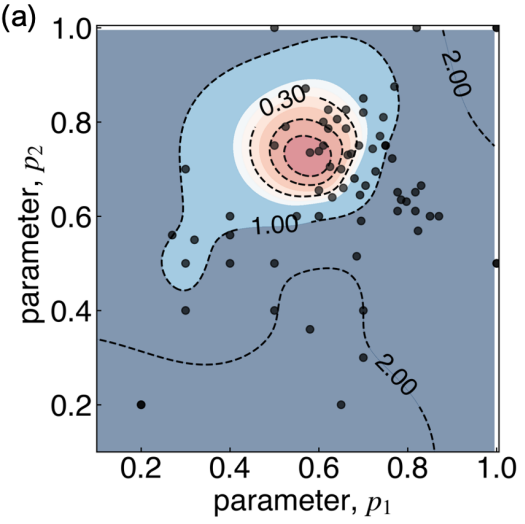

(d)

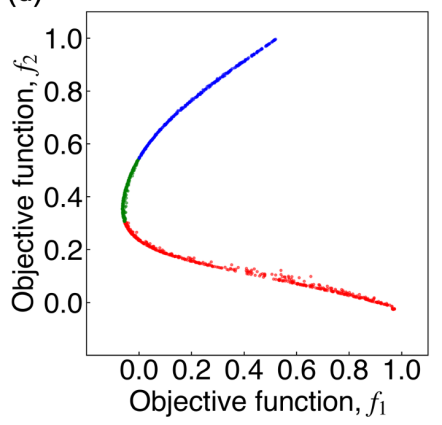

(e) (b)
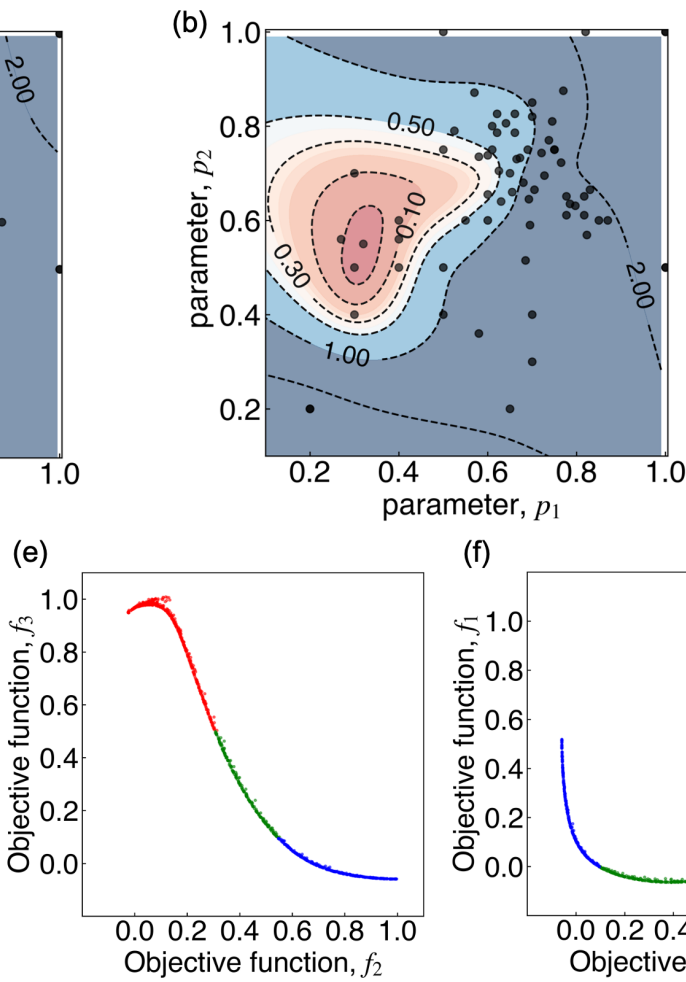

(f)

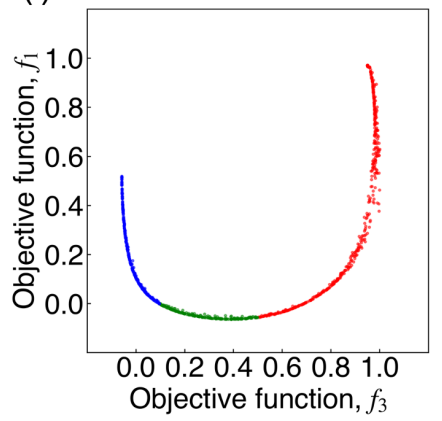

(c)

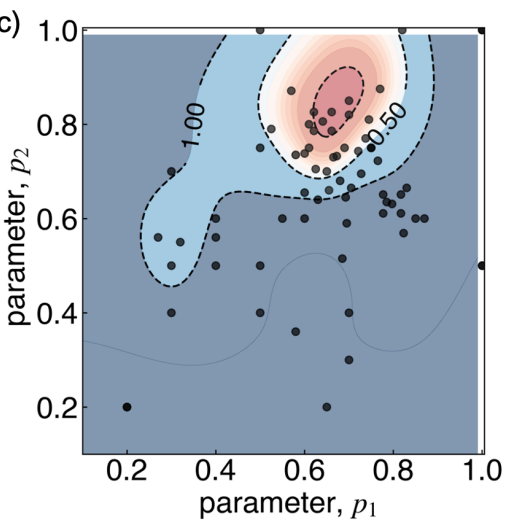

(g)

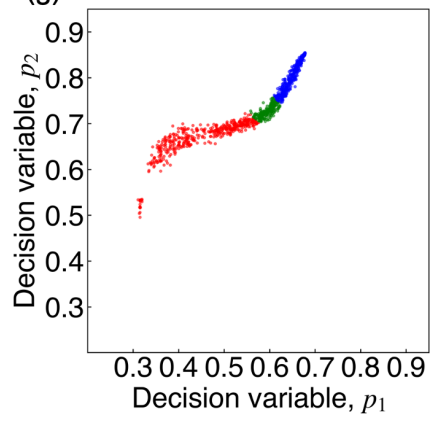

FIG. 2. Response surfaces of objective functions and their Pareto fronts in the case of $p_{1}<p_{2}$. The hyperparameters are set to $\alpha=10$ and $\beta=10^{6}$. Panel (a) represents the response surface of the objective function $f_{1}(\boldsymbol{p})$ of order parameter inferred from the training dataset by GP. Panel (b) represents the response surface of the RDF objective function $f_{2}(\boldsymbol{p})$, and (c) represents that of the objective function $f_{3}(\boldsymbol{p})$ of critical angle, respectively. The training data is represented by black dots and the estimated response surfaces are color coded. The color changes from blue to red as it approaches the optimum region. Panels (d)-(f) show the Pareto fronts for the calibration parameter $\boldsymbol{p}$ : (d) the Pareto front of $f_{1}$ and $f_{2}$ in the objective function space, (e) that of $f_{2}$ and $f_{3}$, (f) that of $f_{3}$ and $f_{1}$. Panel (g) shows the distributions of the Pareto optimal solutions set of $p_{1}$ and $p_{2}$ in the decision variable space. The region of $f_{3}<0.1$ shown by blue dots is referred to as the $\gamma$ optimum region in panels (d)-(g), the region of $f_{3}>0.5$ shown by red dots as the RDF optimum region, and the region of $0.1 \leqq f_{3} \leqq 0.5$ shown by green dots as the balanced region, respectively. The statistics of $p_{1}$ and $p_{2}$ for each region are summarized in Table V.

Their Pareto fronts are shown in Figs. 2(d)-2(g). As shown in Fig. 2(e), $f_{2}$ and $f_{3}$ are inversely correlated. The region of $f_{3}<0.1$ corresponds to $f_{2}>0.6$, and the region of $f_{3}>0.5$ corresponds to $f_{2}<0.3$. We refer to the region of $f_{3}<0.1$ as the $\gamma$ optimum region, which corresponds to the critical angle $\gamma>49.5^{\circ}$. Similarly, we refer to the region of $f_{3}>0.5$ as the RDF optimum region, which corresponds to the critical angle $\gamma<45^{\circ}$. Furthermore, we call the region of $0.1 \leqq f_{3} \leqq$ 0.5 as the balanced region. The statistics of $p_{1}$ and $p_{2}$ for each region are shown in Table $\mathrm{V}$.

The derived response surfaces for $p_{1}>p_{2}$ are shown in Figs. 3(a)-3(c). The optimal ranges of the objective functions $f_{1}, f_{2}$, and $f_{3}$ are around $\left(p_{1}, p_{2}\right)=(0.75,0.6),(0.6,0.4)$, and $(0.8,0.6)$, respectively. Each objective function has a different optimal range and there are trade-offs between them. Their Pareto fronts are shown in Figs. 3(d)-3(g). The statistics of $p_{1}$ and $p_{2}$ for the $\gamma$ optimum, the RDF optimum, and the balanced regions are shown in Table VI.

Next, we examine the hyperparameter dependence of the optimal solutions $\boldsymbol{p}=\left(p_{1}, p_{2}\right)$ to validate the specific values of $\alpha$ and $\beta$. The results are shown in Table VII. The differences of the solutions are $\left|\Delta p_{i}\right| \leqslant 0.02(i=1,2)$ for both $p_{1}<p_{2}$ and $p_{1}>p_{2}$. Since QoIs such as the scalar order parameter $S$, the peak height $G_{z}^{\mathrm{pk}}$ of the density distribution, and the critical angle $\gamma$ are within the target values in the range of $\left|\Delta p_{i}\right|($ cf. Tables XI and XII), the validity of the hyperparameter values of $\alpha=10$ and $\beta=10^{6}$ is confirmed.

The derived Pareto optimal set of CG force fields is summarized as follows. We have two types of intramolecular force fields as shown in Table II, i.e., the flexible alkyl group properties (type WP) and the rigid alkyl group properties (type

TABLE V. Statistics of the $p_{1}$ and $p_{2}$ distributions in the case of $p_{1}<p_{2}$. Mean, standard deviation (s.d.), and quartile in the RDF optimum region $\left(f_{3}<0.1\right)$, the balanced region $\left(0.1 \leqq f_{3} \leqq 0.5\right)$, and the $\gamma$ optimum region $\left(f_{3}>0.5\right)$ in Figs. $2(\mathrm{~d})-2(\mathrm{~g})$ are summarized.

\begin{tabular}{|c|c|c|c|c|c|c|}
\hline & \multicolumn{2}{|c|}{ RDF optimum } & \multicolumn{2}{|c|}{ Balanced } & \multicolumn{2}{|c|}{$\gamma$ optimum } \\
\hline & $p_{1}$ & $p_{2}$ & $p_{1}$ & $p_{2}$ & $p_{1}$ & $p_{2}$ \\
\hline mean & 0.455 & 0.668 & 0.595 & 0.729 & 0.647 & 0.800 \\
\hline s.d. & 0.077 & 0.042 & 0.015 & 0.013 & 0.018 & 0.032 \\
\hline $\min$. & 0.310 & 0.496 & 0.558 & 0.705 & 0.610 & 0.746 \\
\hline $25 \%$ & 0.388 & 0.655 & 0.581 & 0.719 & 0.630 & 0.772 \\
\hline $50 \%$ & 0.467 & 0.682 & 0.597 & 0.728 & 0.646 & 0.797 \\
\hline $75 \%$ & 0.524 & 0.696 & 0.606 & 0.739 & 0.663 & 0.829 \\
\hline $\max$ & 0.576 & 0.722 & 0.622 & 0.757 & 0.678 & 0.855 \\
\hline
\end{tabular}


(a)

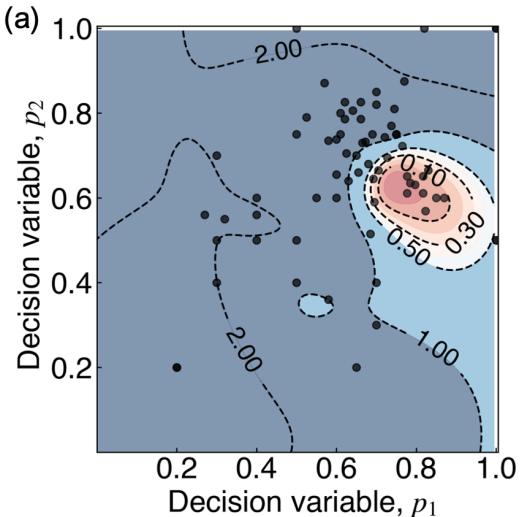

(d)

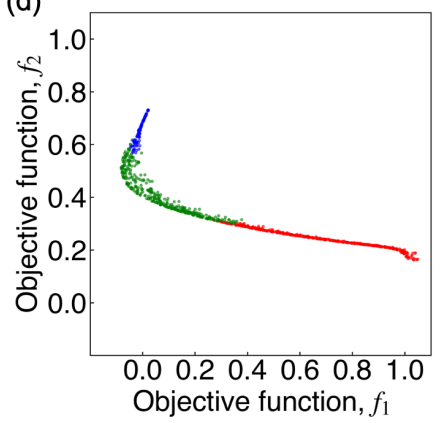

(e) (b)
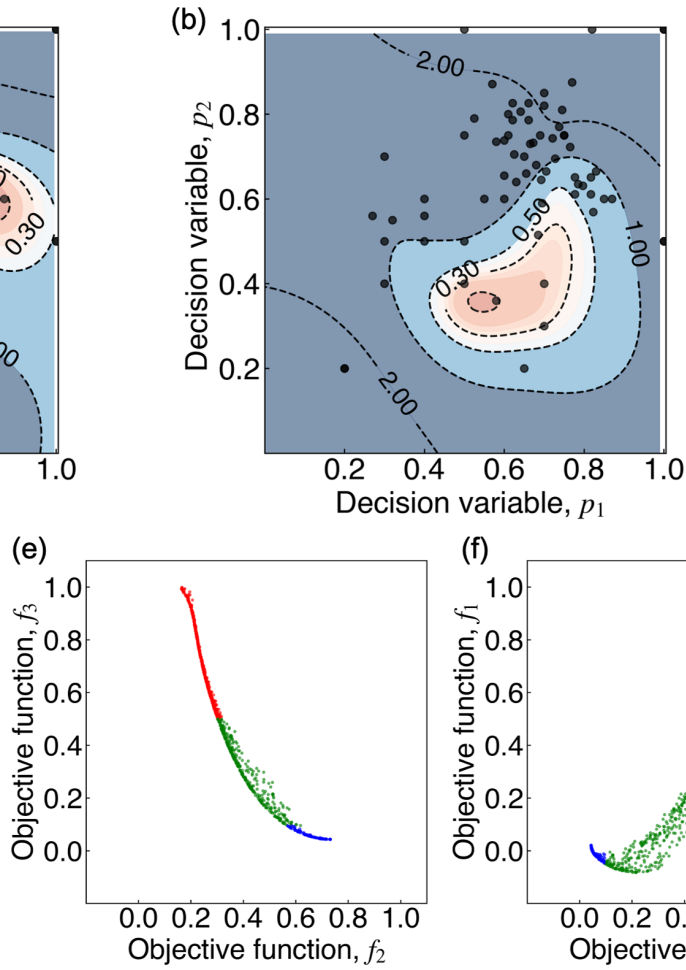

(f)

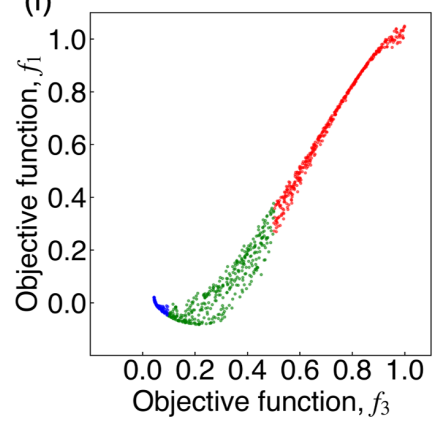

(c)

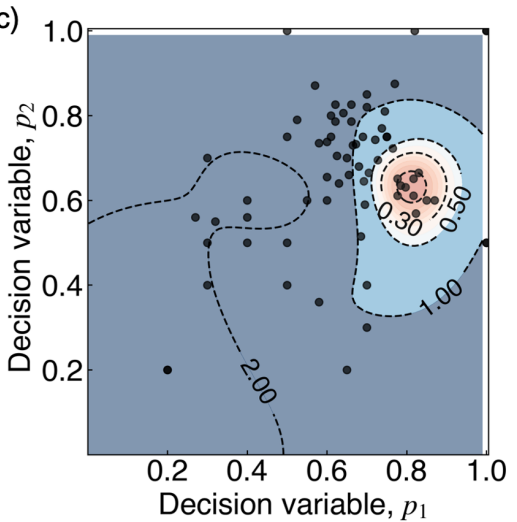

(g)

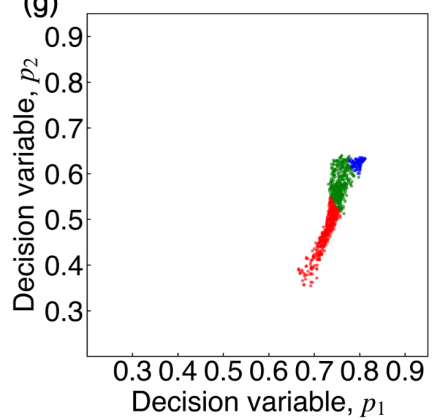

FIG. 3. Response surfaces of objective functions and their Pareto fronts in the case of $p_{1}>p_{2}$. The hyperparameters are set to $\alpha=10$ and $\beta=10^{6}$. Panel (a) represents the response surface of the objective function $f_{1}(\boldsymbol{p})$ of order parameter inferred from the training dataset by GP. Panel (b) represents the response surface of the RDF objective function $f_{2}(\boldsymbol{p})$, and (c) represents that of the objective function $f_{3}(\boldsymbol{p})$ of critical angle, respectively. The training data are represented by black dots and the estimated response surfaces are color coded. The color changes from blue to red as it approaches the optimum region. Panels (d)-(f) show the Pareto fronts for the calibration parameter $p$ : (d) the Pareto front of $f_{1}$ and $f_{2}$ in the objective function space, (e) that of $f_{2}$ and $f_{3}$, and (f) that of $f_{3}$ and $f_{1}$. Panel (g) shows the distributions of the Pareto optimal solutions set of $p_{1}$ and $p_{2}$ in the decision variable space. The region of $f_{3}<0.1$ shown by blue dots is referred to as the $\gamma$ optimum region in panels (d)-(g), the region of $f_{3}>0.5$ shown by red dots as the RDF optimum region, and the region of $0.1 \leqq f_{3} \leqq 0.5$ shown by green dots as the balanced region, respectively. The statistics of $p_{1}$ and $p_{2}$ for each region are summarized in Table VI.

NP). We also have three intermolecular force fields: the force fields for the $\gamma$ optimum region, the balanced region, and the RDF optimum region in each higher head-tail symmetric subdomain $\left(p_{1}<p_{2}\right)$ and lower head-tail symmetric subdomain $\left(p_{1}>p_{2}\right)$, respectively. We combine these force fields to form the candidate models that match the buckling instability of the smectic layer as well as its structural characteristics. Since the flexibility of the alkyl chain plays an important

TABLE VI. Statistics of the $p_{1}$ and $p_{2}$ distributions in the case of $p_{1}>p_{2}$. Mean, standard deviation (s.d.), and quartile in the RDF optimum region $\left(f_{3}<0.1\right)$, the balanced region $\left(0.1 \leqq f_{3} \leqq 0.5\right)$, and the $\gamma$ optimum region $\left(f_{3}>0.5\right)$ in Figs. $3(\mathrm{~d})-3(\mathrm{~g})$ are summarized.

\begin{tabular}{|c|c|c|c|c|c|c|}
\hline & \multicolumn{2}{|c|}{ RDF optimum } & \multicolumn{2}{|c|}{ Balanced } & \multicolumn{2}{|c|}{$\gamma$ optimum } \\
\hline & $p_{1}$ & $p_{2}$ & $p_{1}$ & $p_{2}$ & $p_{1}$ & $p_{2}$ \\
\hline mean & 0.727 & 0.478 & 0.757 & 0.581 & 0.797 & 0.624 \\
\hline s.d. & 0.019 & 0.045 & 0.012 & 0.030 & 0.009 & 0.008 \\
\hline $\min$. & 0.665 & 0.355 & 0.731 & 0.513 & 0.778 & 0.601 \\
\hline $25 \%$ & 0.717 & 0.450 & 0.748 & 0.556 & 0.792 & 0.619 \\
\hline $50 \%$ & 0.732 & 0.487 & 0.756 & 0.577 & 0.798 & 0.627 \\
\hline $75 \%$ & 0.740 & 0.513 & 0.765 & 0.608 & 0.804 & 0.630 \\
\hline $\max$. & 0.757 & 0.550 & 0.798 & 0.640 & 0.811 & 0.636 \\
\hline
\end{tabular}

role in the LC conformation [58], the flexible intramolecular force field, i.e., type WP is employed for the intramolecular force fields. As for the intermolecular force fields, we consider the force field parameters of the $\gamma$ optimum region are suitable for investigating the mechanism of defect generation by the buckling deformation because of the following reason. For the force fields of the balanced and the RDF optimum region, the smectic system collapses and exhibits the complex texture even if the critical collapse angle $\gamma>40^{\circ}$. In contrast, for the force fields of the $\gamma$ optimum region,

TABLE VII. Hyperparameter dependence of $p_{1}$ and $p_{2}$ solutions of the $\gamma$ optimum region in case of $p_{1}<p_{2}$ and $p_{1}>p_{2}$, respectively.

\begin{tabular}{|c|c|c|c|c|c|}
\hline \multicolumn{2}{|c|}{ Hyperparameter } & \multicolumn{2}{|c|}{$p_{1}<p_{2}$} & \multicolumn{2}{|c|}{$p_{1}>p_{2}$} \\
\hline$\alpha$ & $\beta$ & $p_{1}$ & $p_{2}$ & $p_{1}$ & $p_{2}$ \\
\hline 5 & $10^{6}$ & 0.643 & 0.796 & 0.798 & 0.615 \\
\hline 10 & $10^{4}$ & 0.648 & 0.801 & 0.797 & 0.626 \\
\hline 10 & $10^{6}$ & 0.647 & 0.800 & 0.797 & 0.624 \\
\hline 10 & $10^{8}$ & 0.647 & 0.801 & 0.796 & 0.626 \\
\hline 25 & $10^{6}$ & 0.639 & 0.806 & 0.800 & 0.629 \\
\hline 50 & $10^{6}$ & 0.639 & 0.810 & 0.801 & 0.635 \\
\hline
\end{tabular}


TABLE VIII. Parameters of LJ potentials [cf. Eq. (3)] for the intermolecular interactions of model 2. The parameters of type WP in Table II are adopted as the intramolecular interactions [cf. Eq. (2)].

\begin{tabular}{lccccc}
\hline \hline CG-CG & $\begin{array}{c}\sigma \\
(\mathrm{nm})\end{array}$ & $\begin{array}{c}\epsilon \\
\left(\mathrm{kJ} \mathrm{mol}^{-1}\right)\end{array}$ & CG-CG & $\begin{array}{c}\sigma \\
(\mathrm{nm})\end{array}$ & $\begin{array}{c}\epsilon \\
\left(\mathrm{kJ} \mathrm{mol}^{-1}\right)\end{array}$ \\
\hline CN-CN & 0.314 & 2.559 & CN-P3 & 0.360 & 1.980 \\
P3-P3 & 0.468 & 0.946 & CN-C6 & 0.374 & 1.460 \\
C6-C6 & 0.487 & 0.922 & CN-AL & 0.350 & 2.063 \\
AL-AL & 0.377 & 2.074 & P3-C6 & 0.469 & 0.469 \\
& & & P3-AL & 0.433 & 0.555 \\
& & & C6-AL & 0.373 & 0.827 \\
\hline \hline
\end{tabular}

the smectic system collapses with generating the topological defects, which we will describe in the next section in detail. Therefore, we select the force field parameters from the $\gamma$ optimum region in each of $p_{1}<p_{2}$ and $p_{1}>p_{2}$ subdomains. We set $\left(p_{1}, p_{2}\right)=(0.641,0.806)$ and $(0.797,0.631)$ from the optimal range of decision variables $\boldsymbol{p}$ of each subdomain, respectively (cf. Table VII). The force field in $p_{1}<p_{2}$ subdomain shown in Table VIII is the higher head-tail symmetric model. We refer to this model as model 2. The force field in $p_{1}>p_{2}$ subdomain shown in Table IX is the lower head-tail symmetric model. We refer to this model as model 3 . We use the flexible alkyl group (type WP in Table II) as the intramolecular force fields for these models.

\section{RESULTS AND DISCUSSION}

\section{A. Performance of the derived CG models}

Table X shows the performance of models 1, 2, and 3 in terms of the scalar orientational order parameter $S$, the layer critical angle $\gamma$, the peak height $G_{z}^{\mathrm{pk}}$ of the density distribution $G_{z}(z)$ of $\mathrm{CN}$ particles at the position $z$ along the layer normal. We also show the differences in RDF peak heights of C6-C6 particles from the target united-atom model, and the difference of $G_{z}^{\mathrm{pk}}$. Figure 4 shows the density distributions $G_{z}(z)$ of CN particles along $z$ direction for models 1,2 , and 3, respectively. QoIs such as $S, \mathrm{RDF}$, and $G_{z}(z)$ of models 2 and 3 are closer to the target values compared to those of model 1. It can be confirmed that models 2 and 3 reproduce the structural properties of the UA model as well as its order parameter $S$ and the layer critical angle $\gamma$ more precisely than model 1 . We also examine the robustness of the derived parameter sets

TABLE IX. Parameters of LJ potentials [cf. Eq. (3)] for the intermolecular interactions of model 3. The parameters of type WP in Table II are adopted as the intramolecular interactions [cf. Eq. (2)].

\begin{tabular}{lccccc}
\hline \hline CG-CG & $\begin{array}{c}\sigma \\
(\mathrm{nm})\end{array}$ & $\begin{array}{c}\epsilon \\
\left(\mathrm{kJ} \mathrm{mol}^{-1}\right)\end{array}$ & CG-CG & $\begin{array}{c}\sigma \\
(\mathrm{nm})\end{array}$ & $\begin{array}{c}\epsilon \\
\left(\mathrm{kJ} \mathrm{mol}^{-1}\right)\end{array}$ \\
\hline CN-CN & 0.314 & 3.182 & CN-P3 & 0.360 & 2.208 \\
P3-P3 & 0.468 & 0.946 & CN-C6 & 0.374 & 1.628 \\
C6-C6 & 0.487 & 0.922 & CN-AL & 0.350 & 2.035 \\
AL-AL & 0.377 & 1.623 & P3-C6 & 0.469 & 0.469 \\
& & & P3-AL & 0.433 & 0.491 \\
& & & C6-AL & 0.373 & 0.732 \\
\hline \hline
\end{tabular}

TABLE X. Comparison of model characteristics. The scalar orientational order parameter $S$ [cf. Eq. (A1)], the peak height $G_{z}^{\mathrm{pk}}$ of the density distribution, the layer critical angle $\gamma$, the RDF differences $\triangle g_{0}(k),(k=1,2)$ at the first and second peak [cf. Eqs. (6) and (8)], and density distribution difference $\triangle G_{z}(z)$ [cf. Eq. (7)] of models 1 , 2, and 3 (cf. Tables III, VIII, and IX) are summarized.

\begin{tabular}{lcccc}
\hline \hline & Model 1 & Model 2 & Model 3 & References \\
\hline$S$ & 0.758 & 0.698 & 0.661 & $0.6-0.7[50,51]$ \\
$\gamma$ & $60^{\circ}$ & $55^{\circ}$ & $53^{\circ}$ & $50^{\circ}-70^{\circ}[9]$ \\
$G_{z}^{\mathrm{pk}}\left(\mathrm{nm}^{-3}\right)$ & 13.6 & 10.7 & 10.1 & - \\
$\triangle g_{0}(1)$ & 0.462 & 0.131 & 0.111 & - \\
$\triangle g_{0}(2)$ & 0.006 & 0.002 & 0.004 & - \\
$\triangle G_{z}(z)$ & 0.600 & 0.257 & 0.189 & - \\
\hline \hline
\end{tabular}

for models 2 and 3. We examine the variation of QoIs $(S$, $\left.\gamma, G_{z}^{\mathrm{pk}}\right)$ over the neighborhood of the force field parameters of model 2 and model 3 . The range for confirmation is $\left(p_{1}, p_{2}\right)=\left(p_{1}^{\mathrm{c}}-0.02, p_{2}^{\mathrm{c}}-0.02\right)$ to $\left(p_{1}^{\mathrm{c}}+0.02, p_{2}^{\mathrm{c}}+0.02\right)$ in the parameter space of the correction factor, where the center of the range $\left(p_{1}^{\mathrm{c}}, p_{2}^{\mathrm{c}}\right)$ is the correction factor of model 2 or model 3 . The evaluation range has been determined from the optimum values for all hyperparameters in Table VII. Nine points in the evaluation range are selected according to the Latin square design for model 2 and model 3, respectively. As shown in Table XI, the variations of QoIs for model 2 are $S=0.678-0.703$ (0.698), $\gamma=48-57(55)$, and $G_{z}^{\mathrm{pk}}=10.5-$ 11.0 (10.7), where the values in parentheses indicate those of model 2. As shown in Table XII, the variations of QoIs for model 3 are $S=0.651-0.676$ (0.661), $\gamma=48-60$ (53), and $G_{z}^{\mathrm{pk}}=9.7-50.5(10.1)$, where the values in parentheses indicate those of model 3 . The calculated values meet the target $S=0.6-0.7$ and $\gamma=50-70$, and the variation ratio of $G_{z}^{\mathrm{pk}}$ is $\delta G_{z}^{\mathrm{pk}} / G_{z}^{\mathrm{pk}}<0.08$. Thus, the robustness of the force field parameters of models 2 and 3 is validated.

Next, we show that the derived model reproduces the known characteristics of the buckling deformation and the generation of dislocations [9]. We perform the buckling simulations based on the protocol mentioned in Sec. IIC. The systems are compressed in the $x$ direction up to $\varepsilon=0.64$. The simulation results show that they first undergo the sinusoidal, and then the chevron undulation, on the apex of which the curvature concentrates. The layer angle on both sides of the chevron apex are constant and the opposite sign respectively, and it varies smoothly in the narrow region of the chevron apex. When the curvature of the apex reaches the critical angle, the topological defects are generated in the misorientation domain.

We examine the deformation behavior of model 1 with the LJ potential derived by IBI method (cf. Table III) for comparison with model 2 and 3 in the later discussion. The simulation results are shown in Figs. 5 and 6. Figure 5(a) shows the stress-strain curve of the smectic cell compressed along $x$ axis. Figure 5(b) shows the director angle distributions $\theta(x)$ of the cell at the strain $\varepsilon=0.16,0.32,0.48$, and 0.57 , and Fig. 5(c) shows the corresponding distributions $S(x)$ of scalar orientational order parameter, respectively. The $S(x)$ and $\theta(x)$ are derived by diagonalizing the orientational order 
(a)

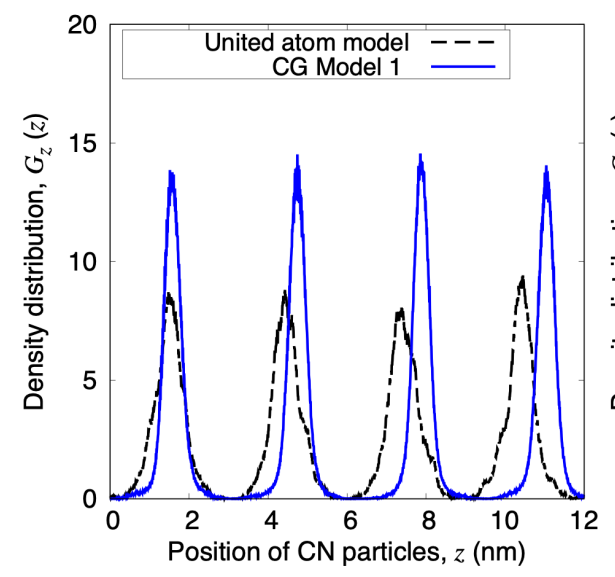

(b)

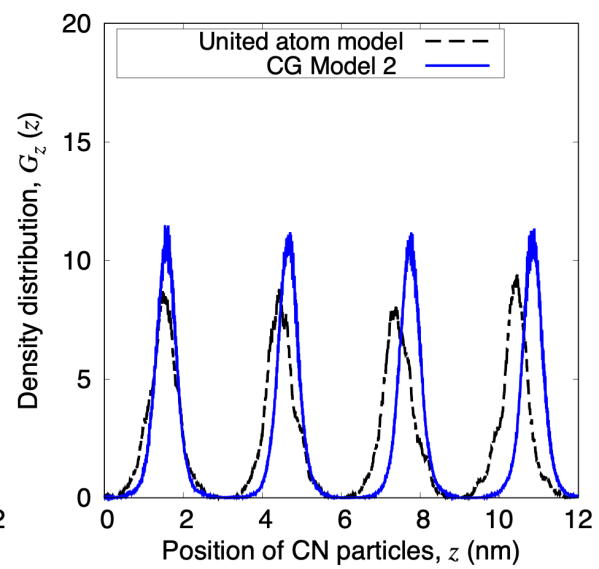

(c)

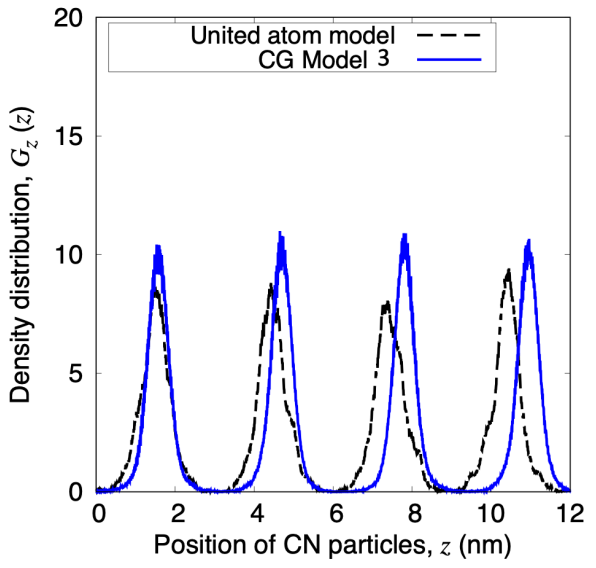

FIG. 4. Equilibrium states of the smectic layers: (a) shows the density distribution of CN particles along $z$ direction of model 1 (cf. Table III), (b) shows that of model 2 (cf. Table VIII), and (c) shows that of model 3 (cf. Table IX). The dashed line is the target distribution of the united-atom model, and the solid line is the distributions of models 1,2 , and 3.

TABLE XI. Variations of QoIs such as the scalar orientational order parameter $S$ [cf. Eq. (A1)], the layer critical angle $\gamma$, and the density distribution $G_{z}^{\mathrm{pk}}$ over the neighborhood of the force field parameters of model 2 (cf. Table VIII). The evaluation range is $\left(p_{1}, p_{2}\right)=\left(p_{1}^{\mathrm{c}}-0.02, p_{2}^{\mathrm{c}}-0.02\right)$ to $\left(p_{1}^{\mathrm{c}}+0.02, p_{2}^{\mathrm{c}}+0.02\right)$ in the parameter space of the correction factor, where the center of the range $\left(p_{1}^{\mathrm{c}}, p_{2}^{\mathrm{c}}\right)$ is the correction factor of model 2. The nine points (models $2 \mathrm{~A}-2 \mathrm{H}$ ) are selected based on the Latin square design and QoIs are calculated from each model. The correction factor $\left(p_{1}, p_{2}\right)$ and the energy parameters $\epsilon_{\mathrm{CN}, \mathrm{CN}}$ and $\epsilon_{\mathrm{AL}, \mathrm{AL}}$ are also shown in the table. The energy parameters $\epsilon_{\mathrm{P} 3, \mathrm{P} 3}$ and $\epsilon_{\mathrm{C} 6, \mathrm{C} 6}$ are the same as those of model 2, and the other energy parameters are calculated with the correction factor $p_{u(i) v(j)}=\sqrt{p_{u(i) u(i)} p_{v(j) v(j)}}$, where $u(i)$ and $v(j)$ are the types of the $i$ th and $j$ th CG particles, respectively. The values of size parameters are the same as those of model 2. The parameters of type WP in Table II are adopted as the intramolecular interactions [cf. Eq. (2)].

\begin{tabular}{|c|c|c|c|c|c|c|c|c|c|c|}
\hline Models & & Model 2 & Model 2A & Model 2B & Model 2C & Model 2D & Model 2E & Model 2F & Model 2G & Model $2 \mathrm{H}$ \\
\hline$p_{1}$ & & 0.641 & 0.661 & 0.621 & 0.641 & 0.641 & 0.661 & 0.621 & 0.661 & 0.621 \\
\hline$p_{2}$ & & 0.806 & 0.806 & 0.806 & 0.826 & 0.786 & 0.826 & 0.786 & 0.786 & 0.826 \\
\hline$\epsilon_{\mathrm{CN}, \mathrm{CN}}$ & $\mathrm{kJ} \mathrm{mol}^{-1}$ & 2.559 & 2.639 & 2.480 & 2.559 & 2.559 & 2.639 & 2.480 & 2.639 & 2.480 \\
\hline$\epsilon_{\mathrm{AL}, \mathrm{AL}}$ & $\mathrm{kJ} \mathrm{mol}^{-1}$ & 2.074 & 2.074 & 2.074 & 2.125 & 2.022 & 2.125 & 2.022 & 2.022 & 2.125 \\
\hline$S$ & & 0.698 & 0.691 & 0.678 & 0.703 & 0.688 & 0.687 & 0.683 & 0.686 & 0.692 \\
\hline$\gamma$ & & $55^{\circ}$ & $57^{\circ}$ & $48^{\circ}$ & $53^{\circ}$ & $50^{\circ}$ & $53^{\circ}$ & $53^{\circ}$ & $55^{\circ}$ & $52^{\circ}$ \\
\hline$G_{z}^{\mathrm{pk}}$ & $\mathrm{nm}^{-3}$ & 10.7 & 11.0 & 10.5 & 11.0 & 10.6 & 10.9 & 10.6 & 10.7 & 10.8 \\
\hline
\end{tabular}

TABLE XII. Variations of QoIs such as the scalar orientational order parameter $S$ [cf. Eq. (A1)], the layer critical angle $\gamma$, and the density distribution $G_{z}^{\mathrm{pk}}$ over the neighborhood of the force field parameters of model 3 (cf. Table IX). The evaluation range is $\left(p_{1}, p_{2}\right)=$ $\left(p_{1}^{\mathrm{c}}-0.02, p_{2}^{\mathrm{c}}-0.02\right)$ to $\left(p_{1}^{\mathrm{c}}+0.02, p_{2}^{\mathrm{c}}+0.02\right)$ in the parameter space of the correction factor, where the center of the range $\left(p_{1}^{\mathrm{c}}, p_{2}^{\mathrm{c}}\right)$ is the correction factor of model 3. The nine points (models $3 \mathrm{~A}-3 \mathrm{H}$ ) are selected based on the Latin square design and QoIs are calculated from each model. The correction factor $\left(p_{1}, p_{2}\right)$ and the energy parameters $\epsilon_{\mathrm{CN}, \mathrm{CN}}$ and $\epsilon_{\mathrm{AL}, \mathrm{AL}}$ are also shown in the table. The energy parameters $\epsilon_{\mathrm{P} 3, \mathrm{P} 3}$ and $\epsilon_{\mathrm{C} 6, \mathrm{C} 6}$ are the same as those of model 3, and the other energy parameters are calculated with the correction factor $p_{u(i) v(j)}=\sqrt{p_{u(i) u(i)} p_{v(j) v(j)}}$, where $u(i)$ and $v(j)$ are the types of the $i$ th and $j$ th CG particles, respectively. The values of size parameters are the same as those of model 3 . The parameters of type WP in Table II are adopted as the intramolecular interactions [cf. Eq. (2)].

\begin{tabular}{|c|c|c|c|c|c|c|c|c|c|c|}
\hline Models & & Model 3 & Model 3A & Model 3B & Model 3C & Model 3D & Model 3E & Model 3F & Model 3G & Model $3 \mathrm{H}$ \\
\hline$p_{1}$ & & 0.797 & 0.797 & 0.797 & 0.817 & 0.777 & 0.817 & 0.777 & 0.817 & 0.777 \\
\hline$p_{2}$ & & 0.631 & 0.651 & 0.611 & 0.631 & 0.631 & 0.651 & 0.611 & 0.611 & 0.651 \\
\hline$\epsilon_{\mathrm{CN}, \mathrm{CN}}$ & $\mathrm{kJ} \mathrm{mol}^{-1}$ & 3.182 & 3.182 & 3.182 & 3.262 & 3.102 & 3.262 & 3.102 & 3.262 & 3.102 \\
\hline$\epsilon_{\mathrm{AL}, \mathrm{AL}}$ & $\mathrm{kJ} \mathrm{mol}^{-1}$ & 1.623 & 1.675 & 1.572 & 1.623 & 1.623 & 1.675 & 1.572 & 1.572 & 1.675 \\
\hline$S$ & & 0.661 & 0.653 & 0.634 & 0.665 & 0.659 & 0.676 & 0.651 & 0.665 & 0.665 \\
\hline$\gamma$ & & $53^{\circ}$ & $53^{\circ}$ & $54^{\circ}$ & $56^{\circ}$ & $55^{\circ}$ & $53^{\circ}$ & $55^{\circ}$ & $60^{\circ}$ & $54^{\circ}$ \\
\hline$G_{z}^{\mathrm{pk}}$ & $\mathrm{nm}^{-3}$ & 10.1 & 10.4 & 9.77 & 10.2 & 10.0 & 10.5 & 9.70 & 10.0 & 10.1 \\
\hline
\end{tabular}


(a)

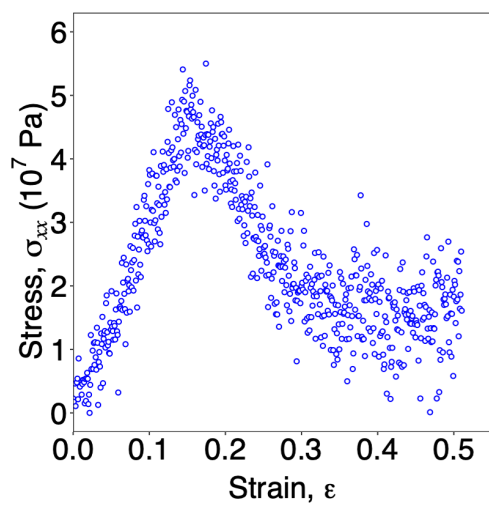

(b)

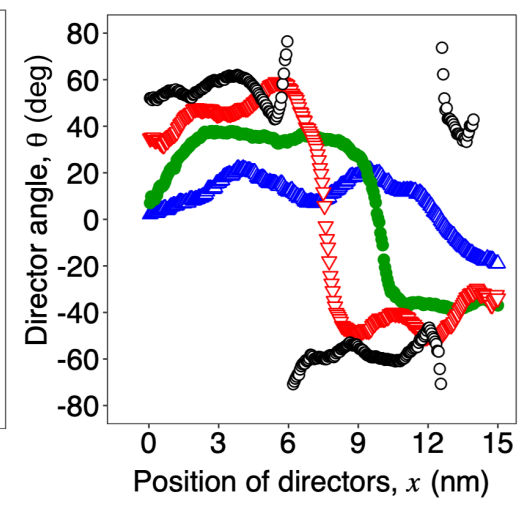

(c)

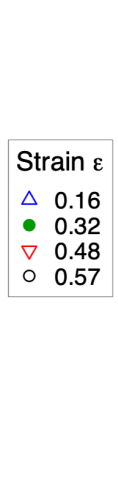

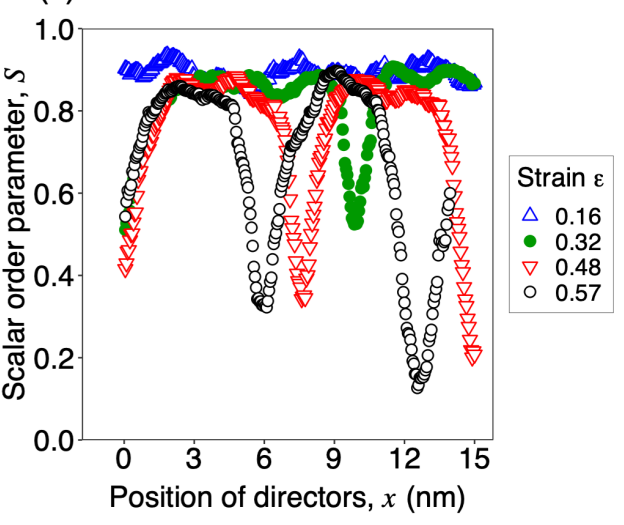

FIG. 5. Deformed states of the smectic layers obtained from model 1 (cf. Table III): Panel (a) shows the stress-strain curve $\left(\sigma_{x x}-\varepsilon\right)$ of the smectic cell compressed along $x$ axis, where $\sigma_{x x}$ is the $x x$ components of the stress tensor, (b) shows the director angle distributions of the cell at the strain $\varepsilon=0.16,0.32,0.48$, and 0.57 , and (c) shows the corresponding distributions of scalar orientational order parameter $S$, respectively. Beyond $\varepsilon=0.16, S$ drops at the apex, and the plastic regime emerges. At the strain $\varepsilon=0.57$ (the critical angle around $\theta=60^{\circ}$ ), dislocations are generated, and the director angle at the apex changes from $0^{\circ}$ to $90^{\circ}$.

tensor $\boldsymbol{Q}$ with Eq. (A1), where the vector $\boldsymbol{d}$ is the molecular long axis between $\mathrm{P} 3-\mathrm{P} 3$ particles, and the ensemble averages of the molecular vectors are calculated at $0.5-\mu \mathrm{m}$ interval along the $x$ axis. We define the director angle $\theta$ as the angle between the plane normal to the layer and $z$ axis in the $x z$ plane, which corresponds to the layer angle in the smectic-A phase as shown in Fig. 1. As the strain increases, the system first undergoes the sinusoidal undulation, and the stress-strain relation of the system belongs to the linear elastic regime, shown in Fig. 5(a). The elasticity in this regime is confirmed by the fact that the system restores a flat shape when the load is removed. Beyond $\varepsilon=0.16$, the system undergoes the chevron undulation and enters the plastic regime. The scalar order parameter $S$ around the apex drops as low as $S=0.4-0.5$ due to the melt of the smectic layer at the apex as shown in Fig. 5(c). At $\varepsilon=0.32$, the director angle is distributed at $\theta \approx 40^{\circ}\left(=\theta_{0}\right)$ on one side of the apex and at $\theta \approx-40^{\circ}\left(=-\theta_{0}\right)$ on the other side as shown in Fig. 5 (b). The director angle changes as $\theta_{0} \rightarrow 0^{\circ} \rightarrow-\theta_{0}$ in the misorientation domain. When the strain reaches $\varepsilon=0.57$, the director angle distribution suddenly changes from $\theta_{0} \rightarrow 0^{\circ} \rightarrow-\theta_{0}$ to $\theta_{0} \rightarrow 90^{\circ} \rightarrow-\theta_{0}$. That is, the director angle changes from parallel to perpendicular to the $z$ axis at $\varepsilon=0.48-0.57$. The critical angle of the layers on both sides of the apex is about $60^{\circ}$ in agreement with the experimental results [9].

So far, we have described the process of defects generation in the real space. However, we can also capture it in the order parameter space (cf. Appendix) to classify the states in the generation of defects. We define the order parameter space $M$ as $M=\left\{S e^{i 2 \theta} \mid \theta \in[0, \pi)\right\}$. Since the director angle $\theta$ takes 0 to $\pi$ due to $\boldsymbol{n}=-\boldsymbol{n}$ equivalence, and $2 \theta$ takes 0 to $2 \pi$, we show $M$ with the polar plot of $(S, 2 \theta)$. Figures 6(a) and 6(b) show the orientational order parameter distributed over a range of $\pm 3 \mu \mathrm{m}$ around chevron apex (a)

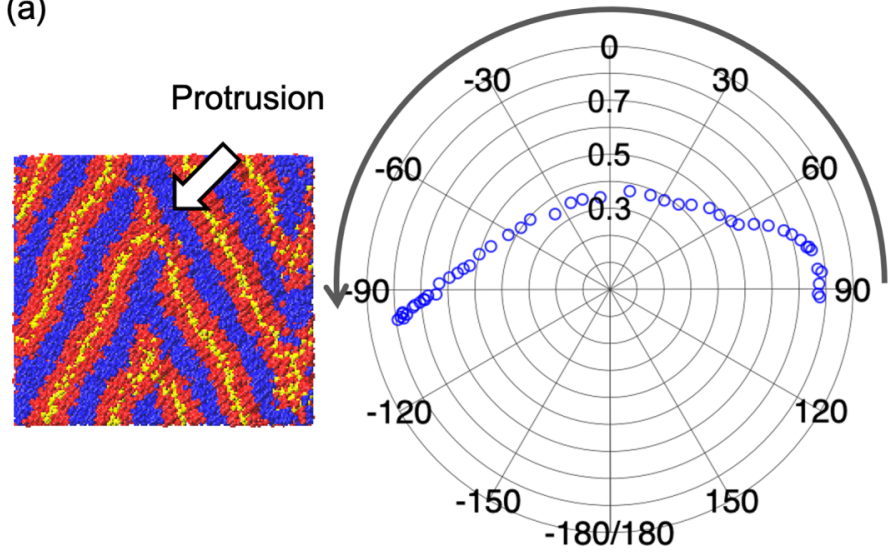

(b)

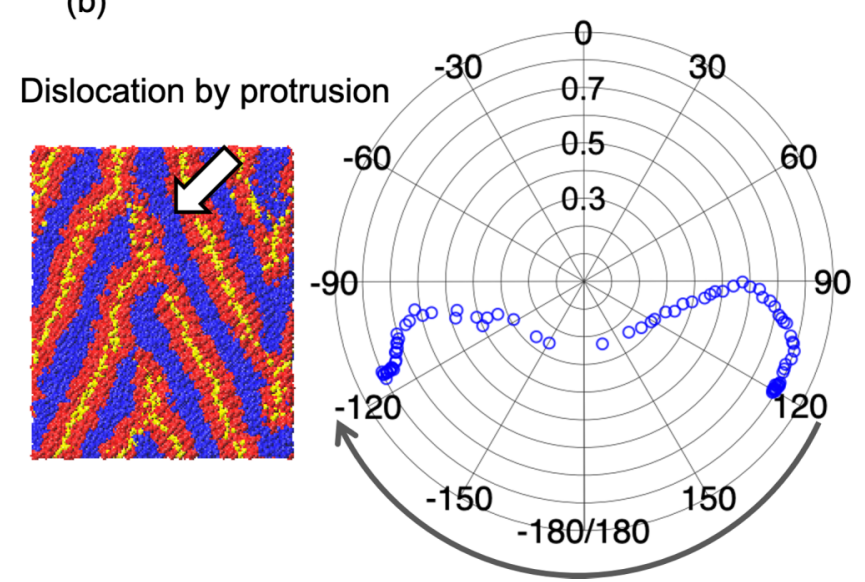

FIG. 6. Deformed states of smectic layers before and after dislocation generation for model 1 (cf. Table III). Panel (a) shows the polar plots of the orientational order parameter $(S, 2 \theta)$ at $\varepsilon=0.48$ and the corresponding snapshot before dislocations are generated. Panel (b) shows the polar plots at $\varepsilon=0.57$ and the corresponding snapshot after dislocations are generated. The radial axis of the polar plots represents the scalar order parameter $S$, and the angular axis represents the angle $2 \theta$, i.e., twice the director angle $\theta$. The $(S, 2 \theta)$ rotates on the upper semicircle of $M$ before dislocation generation, whereas it rotates on the lower semicircle after dislocation generation. 
(a)

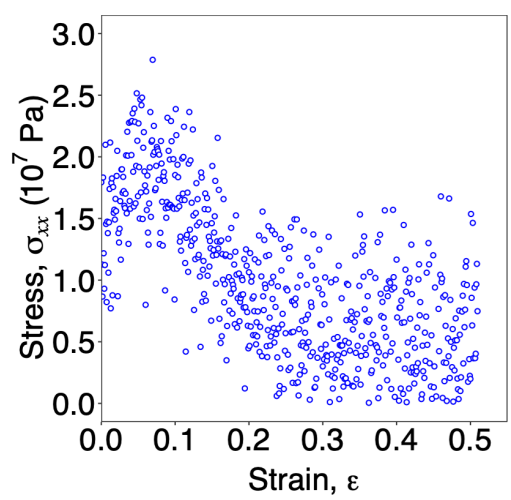

(b)

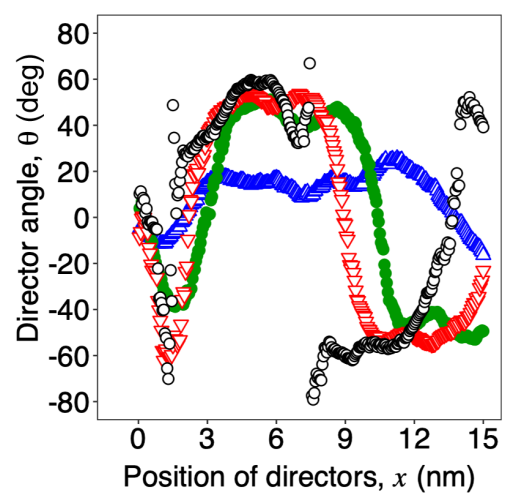

(c)

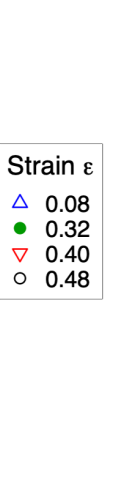

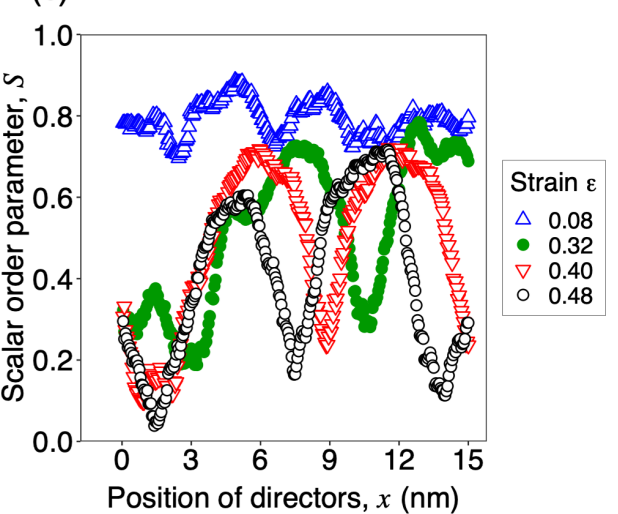

FIG. 7. Deformed states of the smectic layers obtained from Model 2 (cf. Table VIII): (a) shows the stress-strain curve $\left(\sigma_{x x}-\varepsilon\right.$ ) of the smectic cell compressed along $x$ axis, where $\sigma_{x x}$ is the $x x$ components of the stress tensor, (b) shows the director angle distributions at the strain $\varepsilon=0.08,0.32,0.40$, and 0.48 , and (c) shows the corresponding distributions of scalar orientational order parameter $S$, respectively. Beyond $\varepsilon=0.08, S$ drops at the apex, and the plastic regime emerges. At the strain $\varepsilon=0.48$ (the critical angle around $\theta=55^{\circ}$ ), dislocations are generated, and the director angle at the apex changes from $0^{\circ}$ to $90^{\circ}$.

before and after the defect generation, and their corresponding snapshots. Before the generation of defects, the orientational order parameter $(S, 2 \theta)$ rotates counterclockwise on the upper semicircle around the center of $M$. As the strain increases, a protrusion with $-\frac{1}{2}$ disclinations appears and grows at the chevron apex as shown in Fig. 6(a), and $S$ at the apex decreases from $S=0.5$ to 0.3 . Nevertheless, $(S, 2 \theta)$ still rotates counterclockwise on the upper semicircle. At $\varepsilon=0.57$, the protrusion grows and connects to another layer, forming a dislocation by protrusion with $-\frac{1}{2}$ disclination as shown in Fig. 6(b). ( $S, 2 \theta)$ rotates clockwise on the lower semicircle in the opposite direction. Therefore, we can classify the deformation states before and after the dislocation generation as whether the orientational order parameter $(S, 2 \theta)$ rotates counterclockwise in the upper semicircle or clockwise in the lower semicircle. We will explain these results from the topological view point in the next subsection.
Now we compare the deformation behaviors of models 2 and 3 with that of model 1 to confirm that our calibrated models are more precise. Figures 7 and 8 show the results for model 2 where the critical angle is priorized in $p_{1}<p_{2}$ subdomain (cf. Table VIII). In model 2, the transition to the plastic deformation occurs at $\varepsilon=0.08$, and the critical stress is about $\sigma_{x x}^{\mathrm{c}}=2.5 \times 10^{7} \mathrm{~Pa}$, which is smaller than $\sigma_{x x}^{\mathrm{c}}=5 \times 10^{7} \mathrm{~Pa}$ in model 1 as shown in Figs. 5 and 7. Since the unit size of the periodic system in the simulation is about $10 \mathrm{~nm}$, the derived critical stresses of both models are substantially larger than that of $10-\mu \mathrm{m}$-thick cell mentioned in the Introduction. As for the defects, the protrusions are connected to the adjacent layers, and the dislocations by protrusions are formed at $\varepsilon=0.48$ as shown in Fig. 8. These are the same type as the dislocations of model 1 . As for the order parameter $(S, 2 \theta)$ in $M$, it rotates counterclockwise in the upper semicircle at $\varepsilon=0.32$, and rotates clockwise in the lower semicircle at $\varepsilon=0.48$. The direction of rotation is reversed in dislocation generation. (a)

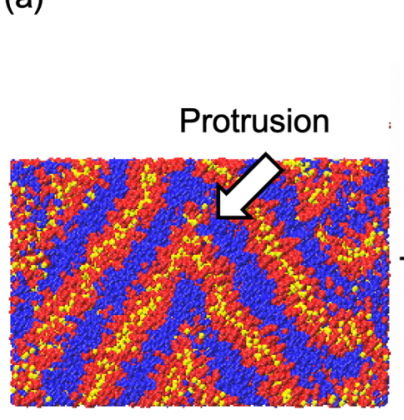

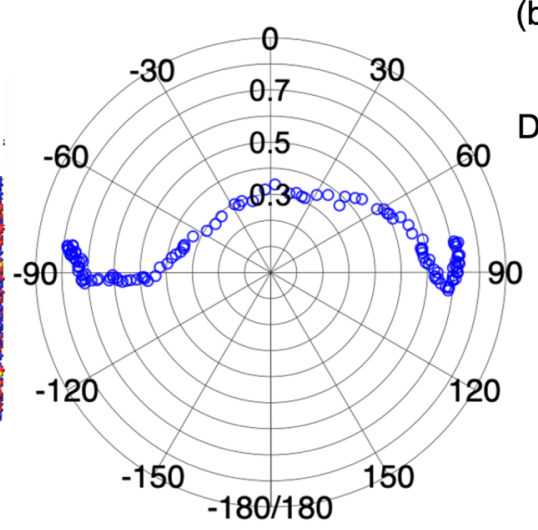

(b)

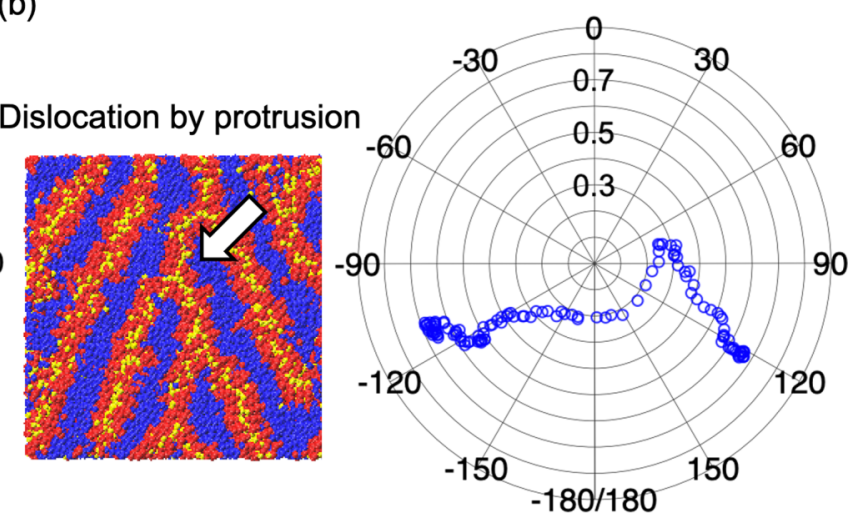

FIG. 8. Deformed states of smectic layers before and after dislocation generation for model 2 (cf. Table VIII). Panel (a) shows the polar plots of the orientational order parameter $(S, 2 \theta)$ at $\varepsilon=0.32$ and the corresponding snapshot before dislocations are generated. Panel (b) shows the polar plots at $\varepsilon=0.48$ for model 2 and the corresponding snapshot after dislocations are generated. The radial axis of the polar plots represents the scalar order parameter $S$, and the angular axis represents the angle $2 \theta$, i.e., twice the director angle $\theta$. The $(S, 2 \theta)$ rotates on the upper semicircle of $M$ before dislocation generation, whereas it rotates on the lower semicircle after dislocation generation. 
(a)

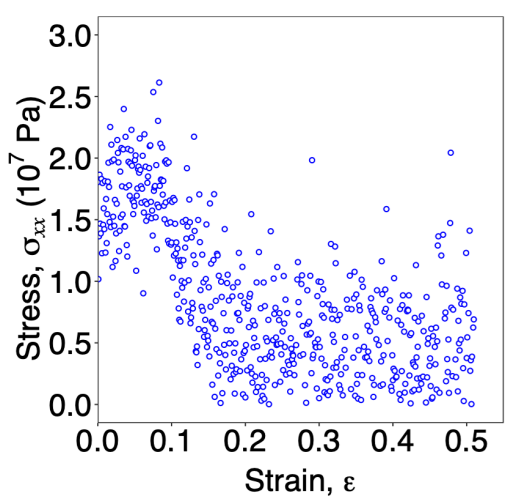

(b)

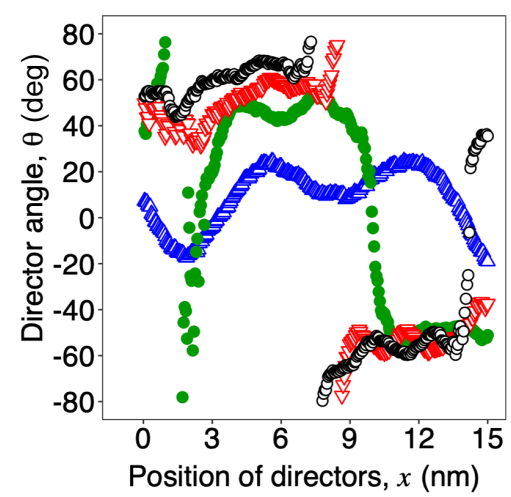

(c)

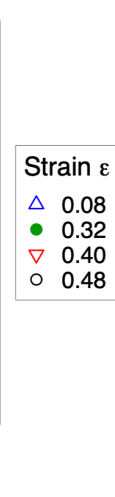

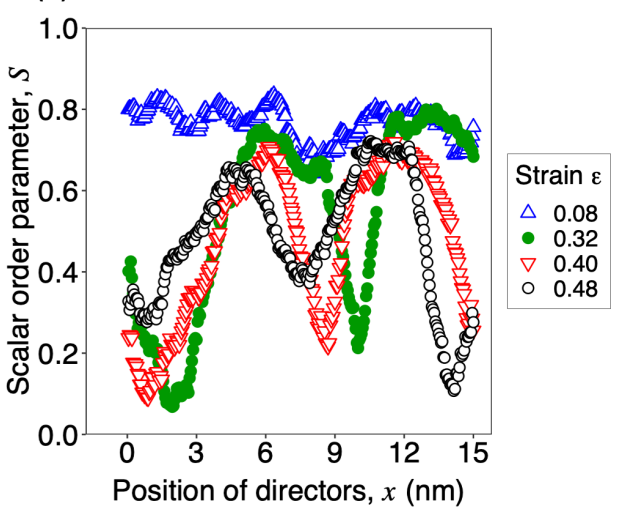

FIG. 9. Deformed states of the smectic layers obtained from model 3 (cf. Table IX): (a) shows the stress-strain curve $\left(\sigma_{x x}-\varepsilon\right)$ of the smectic cell compressed along $x$ axis, where $\sigma_{x x}$ is the $x x$ components of the stress tensor, (b) shows the director angle distributions of the cell at the strain $\varepsilon=0.08,0.32,0.40$, and 0.48 , and (c) shows the corresponding distributions of scalar orientational order parameter $S$, respectively. Beyond $\varepsilon=0.08, S$ drops at the apex, and the plastic regime emerges. At the strain $\varepsilon=0.40$ (the critical angle around $\theta=55^{\circ}$ ), dislocations are generated, and the director angle at the apex changes from $0^{\circ}$ to $90^{\circ}$.

Figures 9 and 10 show the results for model 3, where the critical angle is priorized in $p_{1}>p_{2}$ subdomain (cf. Table IX). The transition occurs at $\varepsilon=0.08$ and the critical stress is about $\sigma_{x x}^{\mathrm{c}}=2.2 \times 10^{7} \mathrm{~Pa}$, which is similar to that of model 2 as shown in Fig. 9. The order parameter $(S, 2 \theta)$ in $M$ rotates counterclockwise in the upper semicircle at $\varepsilon=0.32$, and rotates clockwise in the lower semicircle at $\varepsilon=0.48$ as shown in Fig. 10. The reversal rotation of $(S, \theta)$ after the dislocation generation appears in the same manner as model 2 . However, the dislocation type is different. In model 2, the protrusions grow at the apex, and then the dislocations by protrusion are generated as shown in Fig. 8. This is a typical edge dislocation with a disclination pair for apolar LCs such as $8 \mathrm{CB}$, which is consistent with the experimental results $[9-11,33,59,60]$. In contrast, the layer is cut at the apex, and then the dislocations by layer disconnections are generated in model 3 as shown in Fig. 10. Although the dislocation by layer disconnection has not been observed for apolar LCs, such dislocations is consis- tent with the experimental results for lipid bilayers with strong polarities [59].

In summary, the scalar orientational order parameter $S$ and the density distribution $G_{z}(z)$ of models 2 and 3 are closer to the target values than those of model 1. The transition from linear elastic deformation to plastic deformation is caused by melting of the orientational order at the chevron apex in all of models 1, 2, and 3. However, a defect emerges by the excess of the critical curvature of the layer, i.e., the melt of the translational order around the apex, rather than the critical strain or the melt of the orientational order around the apex. Models 1 and 2 reproduce the generation of the dislocations by protrusions with disclination pairs at the critical angle $\gamma=55^{\circ}$ in agreement with the experimental values of $50^{\circ}-$ $70^{\circ}$ [9], whereas the dislocations by layer disconnections are generated in model 3. Thus, model 2 is the most suitable model to investigate the buckling instability of apolar smectic LCs such as $8 \mathrm{CB}$, as it reproduces the buckling-induced (a)

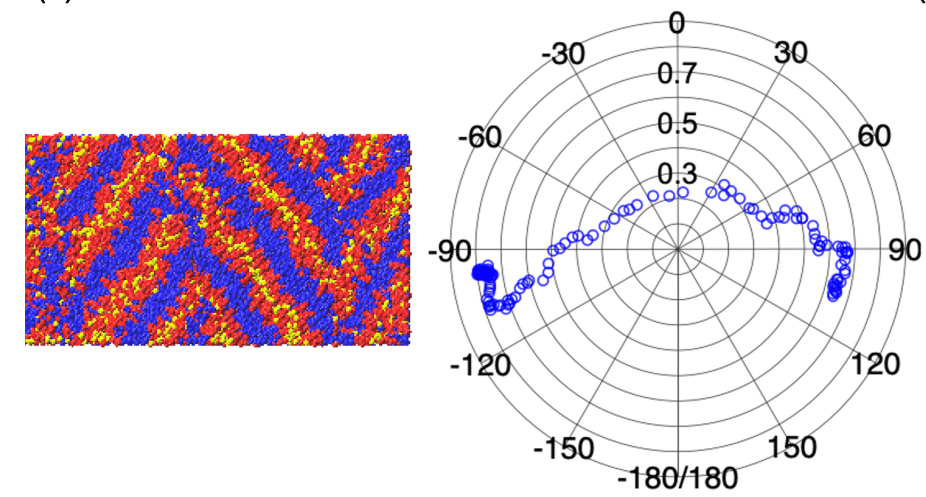

(b)
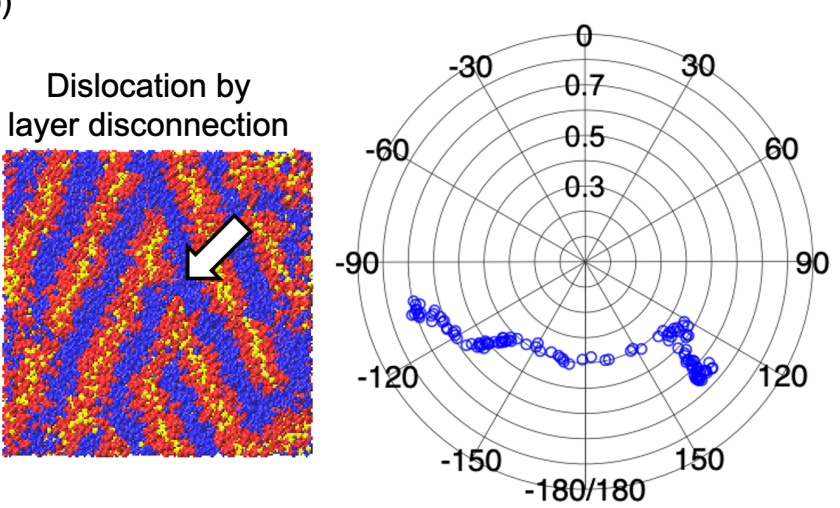

FIG. 10. Deformed states of smectic layers before and after dislocation generation for model 3 (cf. Table IX). Panel (a) shows the polar plots of the orientational order parameter $(S, 2 \theta)$ at $\varepsilon=0.32$ and the corresponding snapshot before dislocations are generated. Panel (b) shows the polar plots at $\varepsilon=0.48$ and the corresponding snapshot after dislocations are generated. The radial axis of the polar plots represents the scalar order parameter $S$, and the angular axis represents the angle $2 \theta$, i.e., twice the director angle $\theta$. The $(S, 2 \theta)$ rotates on the upper semicircle of $M$ before dislocation generation, whereas it rotates on the lower semicircle after dislocation generation. 
(a)

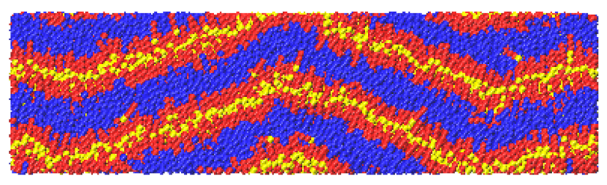

(b)

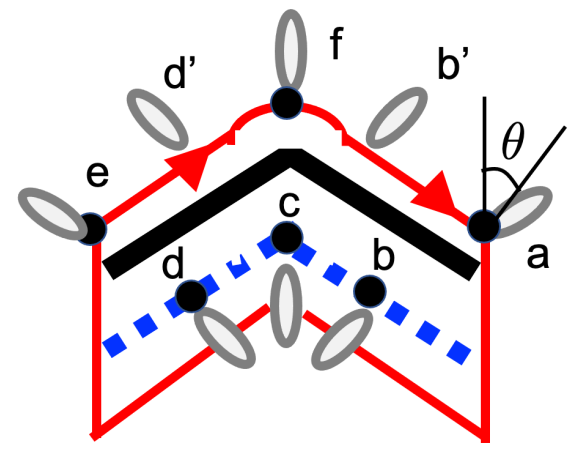

(c)

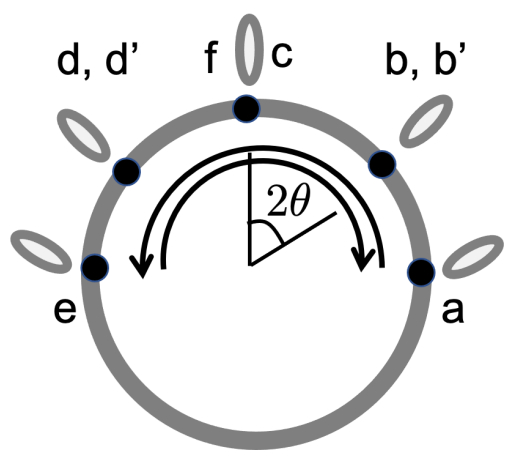

FIG. 11. Defect-free undulated layers of Model 2 (cf. Table VIII). Panel (a) shows a snapshot at $\varepsilon=0.08$, (b) shows the loop around the apex of the buckling layer, where several points a-f are described along the loop, and (c) shows the corresponding loop in the order parameter space $M$, i.e., the circle $S^{1}$ of which the angular axis represents twice the layer angle $2 \theta$. The corresponding map of a-f points are shown in $M=S^{1}$. In panel (b), the thick solid line indicates the density maxima of CN particles, and the dotted line indicates the density maxima of AL particles, which is equivalent to the density minima of $\mathrm{CN}$ particles. The ellipsoids indicate the layer angles pointing in the direction of the layer normal of density maxima of $\mathrm{CN}$ particles and of AL particles.

dislocations with disclinations as well as the QoIs such as $S$ and $G_{z}(z)$. In contrast, model 3 is the suitable model for LCs with large polarities. Comparing models 2 and 3, we find that the dominant factor to control the type of defects is the molecular head-tail symmetry as we will discuss in the next subsection.

\section{B. Relation between the symmetry of force field and the defect type}

As described above, the types of dislocations in model 2 and model 3 are different: the dislocations by protrusions emerge in model 2, whereas the dislocations by layer disconnections emerge in model 3 . The difference between models 2 and 3 is the presence and absence of molecular head-tail symmetry defined by the force field parameters. We discuss the relation between the force field and defect type obtained by CGMD from the viewpoint of LC topology theory [10,3335]. Hereafter, we consider the order parameter space $M=$ $\left\{S e^{i 2 \theta} \mid S=1, \theta \in[0, \pi)\right\}$, i.e., a unit circle. We treat the director $\boldsymbol{n}$ as a unit vector with $\boldsymbol{n}=-\boldsymbol{n}$ equivalence and as $S=1$, according to the LC topology theory.

First, we explain the topology of the defect-free state. Figure 11 shows the undulated layers of model 2 with a loop around a chevron apex, and its mapping to the order parameter space $M$. Figure 11(a) shows a snapshot of the undulation at $\varepsilon=0.08$, Fig. 11(b) shows the loop around the apex of the chevron layer, where several points a-f are described along the loop, and Fig. 11(c) shows the corresponding loop on the order parameter space $M$. In Fig. 11(b), the thick solid line indicates the density maxima of $\mathrm{CN}$ particles, and the dotted line indicates those of $\mathrm{AL}$ particles, which is equivalent to the density minima of $\mathrm{CN}$ particles. The ellipsoids indicate the layer angles pointing the direction of the layer normal of density maxima of $\mathrm{CN}$ particles and those of AL particles. As shown in Fig. 11(b), the layer angles of points $\mathrm{c}$ and $\mathrm{f}$ are both $\theta=0^{\circ}$ before the defect generation. The layer angles rotate counterclockwise in $M$ through points $\mathrm{a} \rightarrow \mathrm{b} \rightarrow \mathrm{c} \rightarrow \mathrm{d}$ on the AL layer, and rotate clockwise through points e $\rightarrow \mathrm{d}^{\prime} \rightarrow \mathrm{f}$ $\rightarrow \mathrm{b}^{\prime} \rightarrow$ a on the CN layer as shown in Fig. 11(c). Therefore, the winding number of the loop, i.e., the topological charge, is zero.

Next, we describe the topology of the dislocations in models 2 and 3 based on Figs. 12 and 13. In model 2, the layer angle is $\theta=90^{\circ}$ at point $\mathrm{f}$ on the $\mathrm{CN}$ layer and $\theta=0^{\circ}$ at point $\mathrm{c}$ on the AL layer. $\theta$ rotates counterclockwise in $M$ at points a $\rightarrow \mathrm{b} \rightarrow \mathrm{c} \rightarrow \mathrm{d}$ on the AL layer, and continue to rotate counterclockwise at points e $\rightarrow \mathrm{f} \rightarrow$ a on the $\mathrm{CN}$ layer. Since the layer angle turns counterclockwise once around the circle, the topological charge of the disclination around the branching point of the $\mathrm{CN}$ layer is $-\frac{1}{2}$. Similarly, the topological charge of the disclination around the gap of the $\mathrm{CN}$ layer is $\frac{1}{2}$. The Burgers vector $\boldsymbol{b}$ of the dislocation is $|\boldsymbol{b}|=5-3=2$. Thus, the dislocation by protrusion is considered as the dislocation of the Burgers vector $|\boldsymbol{b}|=2$ with $\pm \frac{1}{2}$ disclination dipoles. In model 3, the layer angles of point $\mathrm{f}$ on the $\mathrm{CN}$ layer and that of point $\mathrm{c}$ on the $\mathrm{AL}$ layer are both $\theta=90^{\circ}$. The layer angles rotate clockwise in $M$ at points $\mathrm{a} \rightarrow \mathrm{b} \rightarrow \mathrm{c} \rightarrow \mathrm{d}$ on the $\mathrm{CN}$ layer, and rotate counterclockwise for points $\mathrm{e} \rightarrow \mathrm{f} \rightarrow$ a on the AL layer. Therefore, the winding number of the loop, i.e., the topological charge, is zero. The Burgers vector $\boldsymbol{b}$ of the dislocation is $|\boldsymbol{b}|=3-2=1$. Thus, the dislocation by layer disconnection is considered as the dislocation of the Burgers vector $|\boldsymbol{b}|=1$ without disclination.

In summary, since the winding number of dislocations is $-\frac{1}{2}$ around the protrusion in model 2 , the rotational symmetry is broken first, generating the dislocations by protrusions. In contrast, since the winding number of dislocation is zero in model 3, the translational symmetry is broken first, and the layers at the chevron apex are cut off, generating the dislocations by layer disconnections. Thus, the head-tail symmetry is an important factor that determines the nature of the topological defects in deformation. Since each layer in the smectic $\mathrm{SmA}_{\mathrm{d}}$ phase consists of two sublayers of molecules aligned in the opposite direction, the pairs of neighboring molecules belonging to the two different sublayers have head-tail 
(a)

(b)

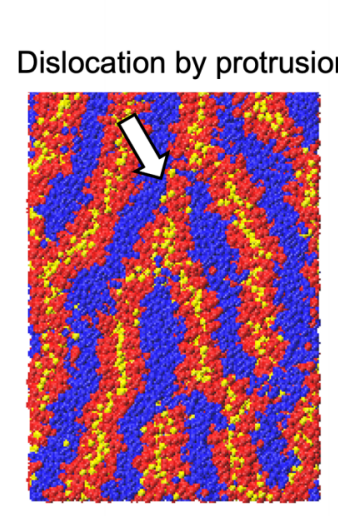

Number of density maxima $=3$

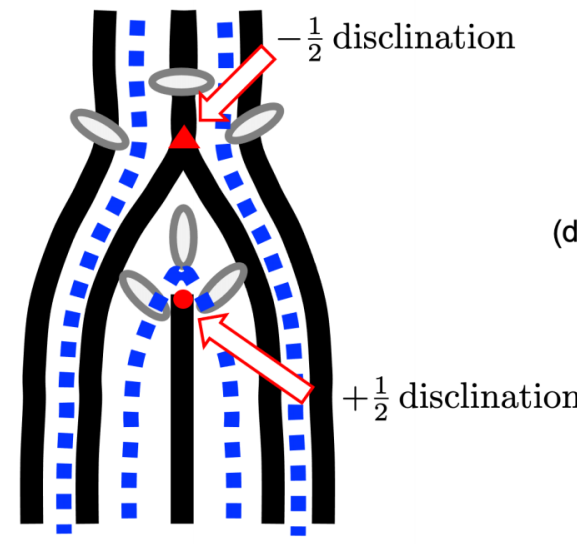

Number of density maxima $=5$ (c)

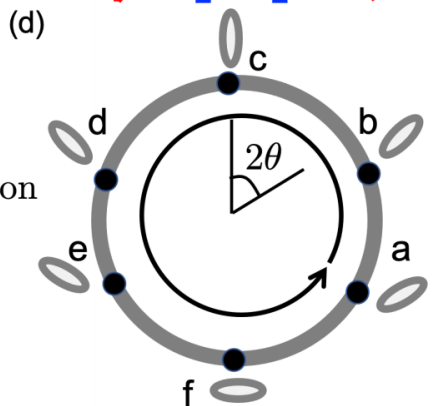

FIG. 12. Disclinations and dislocations of the lamellar system of model 2 (cf. Table VIII). Panel (a) shows the snapshot at $\varepsilon=0.64$, and (b) shows the corresponding schematic of the dipole disclination pair around the edge dislocation: the $-\frac{1}{2}$ disclination located at the branching point of the layer (the triangular point) and the $+\frac{1}{2}$ disclination located between the branching point and the edge of the disconnection layer (the circular point). Panel (c) shows the loop around the $-\frac{1}{2}$ disclination, where several points a-f are described along the loop $\Gamma_{\mathrm{S}}$, and (d) shows the corresponding loop $\Gamma_{\mathrm{M}}$ on the circle $S^{1}$ of the order parameter space $M$. In (b) and (c), the thick solid line indicates the density maxima of $\mathrm{CN}$ particles, and the dotted line indicates the density maxima of AL particles, which is equivalent to the density minima of CN particles. The ellipsoids indicate the layer angles pointing in the direction of the layer normal of density maxima of CN particles and of AL particles. The corresponding map of a-f points are shown in panel (d).

symmetry in the unloaded state. However, large deformations cause the pairs of neighboring molecules belonging to two different sublayers to dissociate at the defect core, and induce the head-tail asymmetry. Therefore, the head-tail symmetry of the constituent single molecule, i.e., the similarity of the energy parameters of $\mathrm{LJ}$ potentials for the terminal groups $\left(\epsilon_{\mathrm{CN}-\mathrm{CN}}, \epsilon_{\mathrm{AL}-\mathrm{AL}}\right)$ determine the types of topological defects. Defining the head-tail symmetry ratio $r=\epsilon_{\mathrm{CN}-\mathrm{CN}} / \epsilon_{\mathrm{AL}-\mathrm{AL}}$, $r=1.23$ and 1.96 in models 2 and 3 , respectively. We summarize the force fields with different symmetry ratios $(r=1.01$, (a)

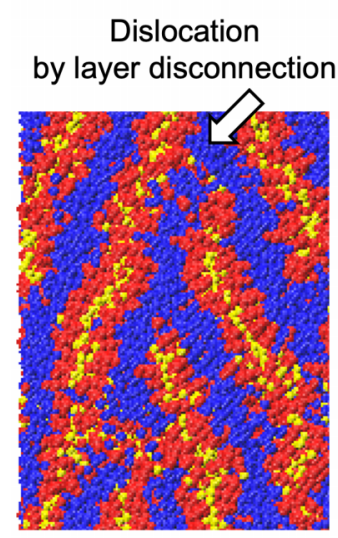

(b)

(c)

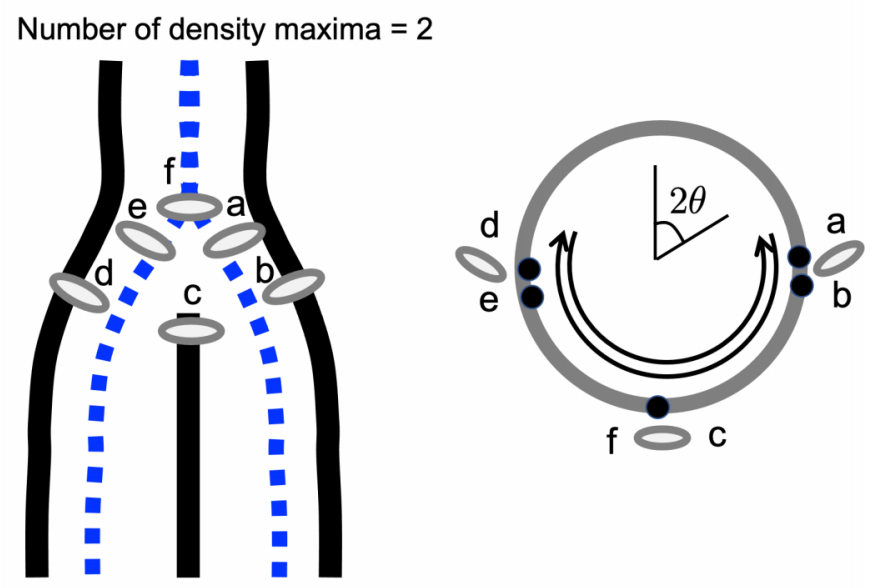

Number of density maxima $=3$

FIG. 13. Disclinations and dislocations of the lamellar system of model 3 (cf. Table IX). Panel (a) shows the snapshot at $\varepsilon=0.64$, (b) shows the corresponding schematic of the dislocation, and (c) shows the corresponding loop $\Gamma_{\mathrm{M}}$ on the circle $S^{1}$ of the order parameter space $M$. In (b), the thick solid line indicates the density maxima of $\mathrm{CN}$ particles, and the dotted line indicates the density maxima of AL particles, which is equivalent to the density minima of $\mathrm{CN}$ particles. The ellipsoids indicate the layer angles pointing in the direction of the layer normal of density maxima of $\mathrm{CN}$ particles and of AL particles. The corresponding map of a-f points are shown in Panel (c). 
TABLE XIII. Models of CG force fields with various head-tail symmetry ratio $r=\epsilon_{\mathrm{CN}-\mathrm{CN}} / \epsilon_{\mathrm{AL}-\mathrm{AL}}$ to study the relation between the molecular symmetry and the types of dislocations. Type $\mathrm{P}$ of dislocations indicates the dislocation with protrusion, and type $\mathrm{D}$ indicates the dislocation by layer disconnection. The correction factor $\left(p_{1}, p_{2}\right)$ and the energy parameters $\epsilon_{\mathrm{CN}, \mathrm{CN}}$ and $\epsilon_{\mathrm{AL}, \mathrm{AL}}$ are also shown in the table. The energy parameters $\epsilon_{\mathrm{P} 3, \mathrm{P} 3}$ and $\epsilon_{\mathrm{C} 6, \mathrm{C} 6}$ are the same as those of models 2 and 3 , and the other energy parameters are calculated with the correction factor $p_{u(i) v(j)}=\sqrt{p_{u(i) u(i)} p_{v(j) v(j)}}$, where $u(i)$ and $v(j)$ are the types of the $i$ th and $j$ th CG particles, respectively. The values of size parameters are the same as those of models 2 and 3. The parameters of type WP in Table II are adopted as the intramolecular interactions [cf. Eq. (2)].

\begin{tabular}{lcccccc}
\hline \hline Models & Model 4A & Model 2 & Model 4B & Model 3 & Model 4C & Model 4D \\
\hline$r$ & 1.01 & 1.23 & 1.85 & 1.96 & 2.06 \\
$p_{1}$ & 0.570 & 0.641 & 0.777 & 0.797 & 0.800 \\
$p_{2}$ & 0.871 & 0.806 & 0.651 & 0.631 & 0.600 \\
$\epsilon_{\mathrm{CN}, \mathrm{CN}}$ & 2.276 & 2.559 & 3.103 & 3.182 & 3.194 \\
$\epsilon_{\mathrm{AL}, \mathrm{AL}}$ & 2.241 & 2.074 & 1.675 & 1.623 & 1.544 \\
Type of dislocations & $\mathrm{kJ} \mathrm{mol}^{-1}$ & $\mathrm{P}$ & $\mathrm{P}$ & $\mathrm{P}$ & $\mathrm{D}$ & $\mathrm{D}$ \\
\hline \hline
\end{tabular}

$1.23,1.85,1.96,2.06$, and 2.32) in Table XIII to compare the types of dislocations. The snapshot of the layer collapse for each model is shown in Fig. 14. It can be confirmed that the rotational symmetry is broken first to induce the dislocation by protrusion for the head-tail symmetric force fields having $r=1.01-1.85$. On the other hand, the translational symmetry is broken first to induce the dislocations by layer disconnections for relatively asymmetric cases having $r=1.96-2.32$. These results are consistent with the experimental results of lipid bilayers with different polarities [59].

\section{CONCLUSION}

We have developed the multiobjective optimization framework based on the Pareto set to derive the CG force field with sufficiently precise buckling characteristics including the molecular details of defect generations. We employ the order parameter and the critical angle of the layer collapse as well as the RDF for the multiobjective functions, and estimated their response surfaces with GP. We divide the search space into subdomains with higher and lower molecular symmetries to find the optimum solution in each subdomain. Based on the response surface of each subdomain, we obtained the Pareto optimal solution using GA, and selected a suitable solution to evaluate the LC deformation. The derived models reproduce the known characteristics of the buckling deformation, the collapse of smectic layers, and the generation of dislocations with dipole disclination pairs, which are consistent with the topological defects theory and experiments. To our knowledge, this is the first study to reproduce the buckling instability and defect generation in smectic LCs by CGMD. Furthermore, we have identified the dominant factors of the molecular force field that determine the nature of buckling-induced topological defects, as well as the factors that determine the deformation characteristics. The transition from linear elastic deformation to plastic deformation is (a)

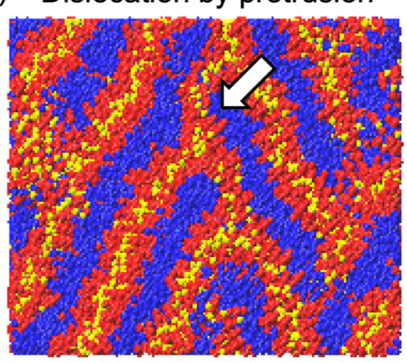

(d)

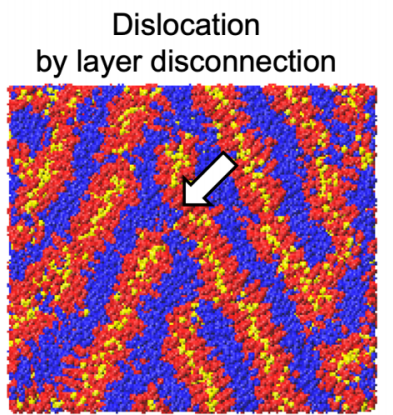

(b)

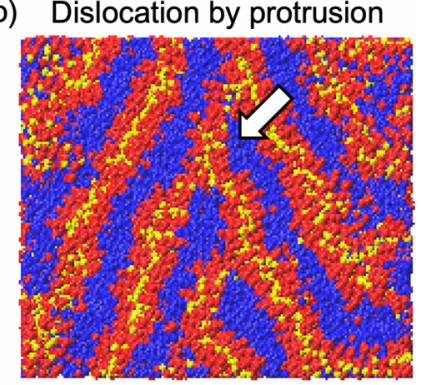

(e)

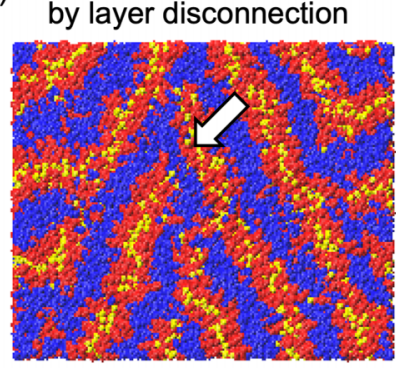

(c) Dislocation by protrusion

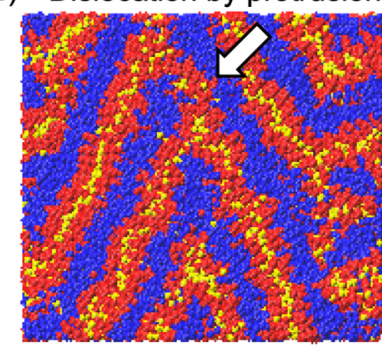

(f)

Dislocation

by layer disconnection

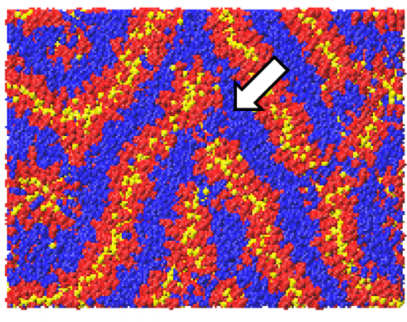

FIG. 14. Snapshots of dislocations of the symmetric and asymmetric force fields (cf. Table XIII): (a) model 4A, (b) model 2, (c) model 4B, (d) model 3, (e) model 4C, and (f) model 4D. It should be noted that the head-tail symmetry ratio $r=1.01,1.23,1.85,1.96,2.06$, and 2.32 for (a), (b), (c), (d), (e), and (f), where $r=\epsilon_{\mathrm{CN}-\mathrm{CN}} / \epsilon_{\mathrm{AL}-\mathrm{AL}}$. 
TABLE XIV. Summary of the derived CG models with respect to their characteristics and force field parameters and the intermolecular potentials. The parameters of type WP in Table II are adopted as the intramolecular interactions [cf. Eq. (2)] for all models.

\begin{tabular}{lcc}
\hline \hline & Characteristics & Force field parameters \\
\hline $\begin{array}{l}\text { Model 1 } \\
\text { Model 2 }\end{array}$ & $\begin{array}{c}\text { Model derived by the IBI method } \\
\text { Model 3 }\end{array}$ & Table III \\
Models 2A-2H & Model that prioritizes the layer critical angle in $p_{1}<p_{2}$ subdomain & Table VIII \\
Models 3A-3H & Models for validating the robustness of model 2 & Table XI \\
Models 4A-4D & Models for validating the robustness of model 3 & Table XII \\
\hline \hline
\end{tabular}

caused by the melt of the orientational order at the chevron apex, while the defect generation is caused by the excess of the critical curvature of the layer, i.e., the melt of the translational order at the apex. The type of the buckling-induced defects depends on the head-tail symmetry of the constituent molecules. The formation of protrusions with disclinations is dominant with the higher head-tail symmetry, whereas the generation of dislocations by layer disconnections is dominant with the lower head-tail symmetry. Finally, we summarize the CG models obtained in this study in Table XIV.

\section{ACKNOWLEDGMENTS}

The computations were performed using Research Center for Computational Science, Okazaki, Japan.

\section{APPENDIX: ORDER PARAMETER AND TOPOLOGICAL DEFECTS}

Liquid crystals exhibit different phases that are characterized by symmetry. Therefore, we need to introduce order parameters to describe the different phases. First, we define an order parameter for a nematic LC. The nematic LC undergoes a phase transition from an isotropic phase to a nematic phase at the phase transition point. The isotropic phase has a high rotational symmetry and is invariant to rotation along any axis. The nematic phase, however, has a lower rotational symmetry. It is invariant to rotation only along a particular axis in which the molecules are aligned. This particular axis is defined as the director $\boldsymbol{n}$, which is a unit vector with head-tail symmetry $(\boldsymbol{n}=-\boldsymbol{n})$. We define the orientational order parameter tensor $\boldsymbol{Q}$ of second-rank moments for the uniaxial nematic phase [61] as

$$
\boldsymbol{Q}=\left\langle\boldsymbol{d} \otimes \boldsymbol{d}-\frac{1}{3} \boldsymbol{I}\right\rangle=S\left(\boldsymbol{n} \otimes \boldsymbol{n}-\frac{1}{3} \boldsymbol{I}\right),
$$

where $\langle x\rangle$ represents the ensemble average of $x$, and $\boldsymbol{d}$ represents the molecular principal axis. The scalar orientational order parameter $S$ representing the strength of the anisotropy can be obtained from the maximum eigenvalue of this orientational order tensor $\boldsymbol{Q}$, and the director $\boldsymbol{n}$ can be calculated from the corresponding eigenvector. Symmetry and topology of the director field can be well described in the order parameter space [10]. The directors $\boldsymbol{n}$ attached to each point $x$ in the real space can take any points $(\theta, \phi)$ in the hemisphere $M=S^{2} / \mathbb{Z}_{2}$ in case of the isotropic phase, where $S^{2}$ represents two-dimensional sphere (2-sphere) and $\mathbb{Z}_{2}$ represents the head-tail symmetry group. When a phase transition occurs, the director $\boldsymbol{n}$ is fixed at a single point on the hemisphere $M$. We refer to the manifold $M$ as the order parameter space.
The order parameter space $M$ for a three-dimensional nematic LC is a hemisphere $S^{2} / \mathbb{Z}_{2}$ with antipodal points identified due to $\boldsymbol{n}=-\boldsymbol{n}$ equivalence. It is a semicircle $S^{1} / \mathbb{Z}_{2}$ for a two-dimensional nematic LC.

Next, we define an order parameter of the smectic phase. The smectic LC shows a lamellar structure with stacks of two-dimensional liquids. Its translational symmetry is one-dimensional. The simplest smectic phase is smectic-A, with an average molecular orientation perpendicular to the layer. Because it is periodic in one dimension, the plane smectic structure is characterized by a sinusoidal density wave $\rho(z)$ with the wave number $q_{1}=2 \pi / a$, where $a$ is the layer spacing and $z$ is the coordinates of the layer normal, i.e., the axis along the director $\boldsymbol{n}$. The density of the undulated smectic system is described as

$$
\rho(z)=\rho_{0}+\rho_{1} \operatorname{Re} \exp \left\{i q_{1}(z-u)\right\} .
$$

where $u$ represents the Eulerian displacement. Therefore, the translational order parameter $\Phi_{\text {tr }}$ of the smectic-A phase is defined as $\Phi_{\mathrm{tr}}=\rho_{1} e^{-i q_{1} u}$. The phase factor of the translational order parameter $\Phi_{\mathrm{tr}}$ is described as $q_{1} u \in[0,2 \pi]$. And the orientational order parameter is the director $\boldsymbol{n}$, whose phase factor is described as $\theta \in[0, \pi]$ for a two-dimensional smectic LC. Therefore, the order parameter space $M$ is the semidirect product of these two order parameter space, described as $M=S^{1} \rtimes S^{1} / \mathbb{Z}_{2}$.

Next, we describe the LC topological defects. If several subdomains with different values of order parameters coexist, then the director misorientation appears at the boundaries, generating the topological defects. There are two types of defects in smectic LCs: dislocation caused by a translational symmetry breaking and disclination caused by a rotational symmetry breaking. These defects can be classified by the topological charge $m$ for dislocations and $l$ for disclinations. For the two-dimensional smectic LC, they are scalars defined as $m=\oint_{\Gamma} \nabla u \cdot d \hat{\boldsymbol{r}}$, and $l=\oint_{\Gamma} \nabla \theta \cdot d \hat{\boldsymbol{r}}$, where $\Gamma$ is a closed loop around the defect and $\hat{\boldsymbol{r}}=\boldsymbol{r} /|\boldsymbol{r}|$. The topological charges $m$ and $l$ correspond to the number of the loop that moves on the translational or orientational order parameter space $M . m$ of dislocations takes an integer value corresponding to the Burgers vector in the loop, whereas $l$ of disclinations takes a half integer value as the orientational order parameter space is a hemisphere or a semicircle. Defects with different topological charges cannot exchange with each other. Therefore, disclinations and dislocations cannot transition to a defectfree state whose topological charge is zero.

In the main text, we only consider the orientational order parameter space, and define the order parameter space $M=$ 
$\left\{S e^{i 2 \theta} \mid \theta \in[0, \pi)\right\}$. Thus, $M$ is the circle $M=S^{1}$, which is the double covering of $S^{1} / \mathbb{Z}_{2}$ mathematically. As for transla- tional symmetry breaking, we consider the Burgers vector in real space.
[1] W. H. de Jeu, B. I. Ostrovskii, and A. N. Shalaginov, Structure and fluctuations of smectic membranes, Rev. Mod. Phys. 75, 181 (2003).

[2] K. Nickmans, J. Murphy, B. de Waal, P. Leclre, J. Doise, R. Gronheid, D. J Broer, and A. Schenning, Sub-5 nm patterning by directed self-assembly of oligo(dimethylsiloxane) liquid crystal thin films, Adv. Mater. 28, 10068 (2016).

[3] D. K. Yoon, M. C. Choi, Y. H. Kim, M. W. Kim, O. D. Lavrentovich, and H.-t. Jung, Internal structure visualization and lithographic use of periodic toroidal holes in liquid crystals, Nat. Mater. 6, 866 (2007).

[4] N. Gheorghiu, J. L. West, A. Glushchenko, and M. Mitrokhin, Patterned field induced polymer walls for smectic a bistable flexible displays, Appl. Phys. Lett. 88, 263511 (2006).

[5] H. Iino and J.-i. Hanna, Liquid crystalline organic semiconductors for organic transistor applications, Polym. J. 49, 23 (2016).

[6] K. Kishimoto, M. Yoshio, T. Mukai, M. Yoshizawa, H. Ohno, and T. Kato, Nanostructured anisotropic ion-conductive films, J. Am. Chem. Soc. 125, 3196 (2003).

[7] J. Toner, Renormalization-group treatment of the dislocation loop model of the smectic-a-nematic transition, Phys. Rev. B 26, 462 (1982).

[8] J. M. Kosterlitz and D. J. Thouless, Ordering, metastability and phase transitions in two-dimensional systems, J. Phys. C 6, 1181 (1973).

[9] J. Jeong and M. W. Kim, Confinement-Induced Transition of Topological Defects in Smectic Liquid Crystals: From a Point to a Line and Pearls, Phys. Rev. Lett. 108, 207802 (2012).

[10] M. Kleman and O. D. Laverntovich, Soft Matter Physics: An Introduction (Springer Science \& Business Media, New York, 2007).

[11] I. I. Smalyukh and O. D. Lavrentovich, Three-dimensional director structures of defects in grandjean-cano wedges of cholesteric liquid crystals studied by fluorescence confocal polarizing microscopy, Phys. Rev. E 66, 051703 (2002).

[12] M. Rahimi, H. Ramezani-Dakhel, R. Zhang, A. RamirezHernandez, N. L. Abbott, and J. J. de Pablo, Segregation of liquid crystal mixtures in topological defects, Nat. Commun. 8, 15064 (2017).

[13] I. Hanasaki, J. H. Walther, S. Kawano, and P. Koumoutsakos, Coarse-grained molecular dynamics simulations of shearinduced instabilities of lipid bilayer membranes in water, Phys. Rev. E 82, 051602 (2010).

[14] G. A. Voth, Coarse-graining of Condensed Phase and Biomolecular Systems (CRC Press, Boca Raton, FL, 2008).

[15] A. C. Callan-Jones, R. A. Pelcovits, V. A. Slavin, S. Zhang, D. H. Laidlaw, and G. B. Loriot, Simulation and visualization of topological defects in nematic liquid crystals, Phys. Rev. E 74, 061701 (2006).

[16] T. Soddemann, G. K. Auernhammer, H. Guo, B. Dünweg, and K. Kremer, Shear-induced undulation of smectic-a: Molecular dynamics simulations vs. analytical theory, Eur. Phys. J. E 13, 141 (2004).
[17] B. Mukherjee, L. Delle Site, K. Kremer, and C. Peter, Derivation of coarse grained models for multiscale simulation of liquid crystalline phase transitions, J. Phys. Chem. B 116, 8474 (2012).

[18] C. Blanc, C. Meyer, S. Asnacios, M. Kleman, I. Lelidis, and J.-L. Martin, Do lamellar liquid crystals flow like solids? Philos. Mag. Lett. 85, 641 (2005).

[19] N. A. Clark and R. B. Meyer, Strain-induced instability of monodomain smectic a and cholesteric liquid crystals, Appl. Phys. Lett. 22, 493 (1973).

[20] M. Delaye, R. Ribotta, and G. Durand, Buckling instability of the layers in a smectic-a liquid crystal, Phys. Lett. A 44, 139 (1973).

[21] P. Oswald and G. Poy, Dislocations dynamics during the nonlinear creep of a homeotropic sample of smectic-a liquid crystal, Eur. Phys. J. E 41, 73 (2018).

[22] H. Bei, Y. F. Gao, S. Shim, E. P. George, and G. M. Pharr, Strength differences arising from homogeneous versus heterogeneous dislocation nucleation, Phys. Rev. B 77, 060103(R) (2008).

[23] A. Jaramillo-Botero, S. Naserifar, and W. A. Goddard III, General multiobjective force field optimization framework, with application to reactive force fields for silicon carbide, J. Chem. Theory Comput. 10, 1426 (2014).

[24] A. Mishra, S. Hong, P. Rajak, C. Sheng, K.-i. Nomura, R. K. Kalia, A. Nakano, and P. Vashishta, Multiobjective genetic training and uncertainty quantification of reactive force fields, npj Comput. Mater. 4, 42 (2018).

[25] K. Deb, A. Pratap, S. Agarwal, and T. Meyarivan, A fast and elitist multiobjective genetic algorithm: NSGA-II, IEEE Transactions on Evolutionary Computation 6, 182 (2002).

[26] F. Leonarski, F. Trovato, V. Tozzini, A. Les, and J. Trylska, Evolutionary algorithm in the optimization of a coarse-grained force field, J. Chem. Theory Comput. 9, 4874 (2013).

[27] C. A. C. Coello, G. B. Lamont, D. A. Van Veldhuizen et al., Evolutionary Algorithms for Solving Multi-objective Problems, Vol. 5 (Springer, Berlin, 2007).

[28] P. Angelikopoulos, C. Papadimitriou, and P. Koumoutsakos, Bayesian uncertainty quantification and propagation in molecular dynamics simulations: A high performance computing framework, J. Chem. Phys. 137, 144103 (2012).

[29] K. Farrell, J. T. Oden, and D. Faghihi, A bayesian framework for adaptive selection, calibration, and validation of coarsegrained models of atomistic systems, J. Comput. Phys. 295, 189 (2015).

[30] S. Wu, P. Angelikopoulos, G. Tauriello, C. Papadimitriou, and P. Koumoutsakos, Fusing heterogeneous data for the calibration of molecular dynamics force fields using hierarchical bayesian models, J. Chem. Phys. 145, 244112 (2016).

[31] A. M. Gopakumar, P. V. Balachandran, D. Xue, J. E. Gubernatis, and T. Lookman, Multi-objective optimization for materials discovery via adaptive design, Sci. Rep. 8, 3738 (2018). 
[32] A. Krishnamoorthy, A. Mishra, N. Grabar, N. Baradwaj, R. Kalia, A. Nakano, and P. Vashishta, Evolutionary multiobjective optimization and pareto-frontal uncertainty quantification of interatomic forcefields for thermal conductivity simulations, Comput. Phys. Commun. 254, 107337 (2020).

[33] M. V. Kurik and O. Lavrentovich, Defects in liquid crystals: Homotopy theory and experimental studies, Sov. Phys. Usp. 31, 196 (1988).

[34] N. D. Mermin, The topological theory of defects in ordered media, Rev. Mod. Phys. 51, 591 (1979).

[35] G. Toulouse and M. Kléman, Principles of a classification of defects in ordered media, J. Phys. Lett. 37, 149 (1976).

[36] G. Tiberio, L. Muccioli, R. Berardi, and C. Zannoni, Towards in silico liquid crystals. realistic transition temperatures and physical properties for n-cyanobiphenyls via molecular dynamics simulations, Chem. Phys. Chem. 10, 125 (2009).

[37] J. Wang, R. M. Wolf, J. W. Caldwell, P. A. Kollman, and D. A. Case, Development and testing of a general amber force field, J. Comput. Chem. 25, 1157 (2004).

[38] N. J. Boyd and M. R. Wilson, Optimization of the gaff force field to describe liquid crystal molecules: The path to a dramatic improvement in transition temperature predictions, Phys. Chem. Chem. Phys. 17, 24851 (2015).

[39] A. Jakalian, B. L. Bush, D. B. Jack, and C. I. Bayly, Fast, efficient generation of high-quality atomic charges. am1-bcc model: I. Method, J. Comput. Chem. 21, 132 (2000).

[40] A. Jakalian, D. B. Jack, and C. I. Bayly, Fast, efficient generation of high-quality atomic charges. am1-bcc model: II. Parameterization and validation, J. Comput. Chem. 23, 1623 (2002).

[41] B. Hess, C. Kutzner, D. Van Der Spoel, and E. Lindahl, Gromacs 4: Algorithms for highly efficient, load-balanced, and scalable molecular simulation, J. Chem. Theory Comput. 4, 435 (2008).

[42] H. J. Berendsen, J. v. Postma, W. F. van Gunsteren, A. DiNola, and J. R. Haak, Molecular dynamics with coupling to an external bath, J. Chem. Phys. 81, 3684 (1984).

[43] D. Tuber and C. Borczyskowski, Single molecule studies on dynamics in liquid crystals, Int. J. Mol. Sci. 14, 19506 (2013).

[44] B. Schulz, M. G. Mazza, and C. Bahr, Single-molecule diffusion in freely suspended smectic films, Phys. Rev. E 90, 040501(R) (2014).

[45] B. Schulz, D. Täuber, J. Schuster, T. Baumgärtel, and C. von Borczyskowski, Influence of mesoscopic structures on single molecule dynamics in thin smectic liquid crystal films, Soft Matter 7, 7431 (2011).

[46] M. Saito, J. Yamamoto, R. Masuda, M. Kurokuzu, Y. Onodera, Y. Yoda, and M. Seto, Direct observation of interlayer molecular translational motion in a smectic phase and determination of the layer order parameter, Phys. Rev. Research 1, 012008(R) (2019).
[47] K. Wang, M. Jirka, P. Rai, R. J. Twieg, T. Szilvási, H. Yu, N. L. Abbott, and M. Mavrikakis, Synthesis and properties of hydroxy tail-terminated cyanobiphenyl liquid crystals, Liq. Cryst. 46, 397 (2019).

[48] F. Eikelschulte, S. Y. Yakovenko, D. Paschek, and A. Geiger, Electrostatic properties of cyano-containing mesogens, Liq. Cryst. 27, 1137 (2000)

[49] C. J. Adam, S. J. Clark, G. J. Ackland, and J. Crain, Conformation-dependent dipoles of liquid crystal molecules and fragments from first principles, Phys. Rev. E 55, 5641 (1997).

[50] M. F. Palermo, A. Pizzirusso, L. Muccioli, and C. Zannoni, An atomistic description of the nematic and smectic phases of 4-n-octyl-4' cyanobiphenyl (8cb), J. Chem. Phys. 138, 204901 (2013).

[51] R. G. Horn, Refractive indices and order parameters of two liquid crystals, J. Phys. 39, 105 (1978).

[52] S. Sharma, K. Neupane, A. Adorjan, A. R. Baldwin, and S. Sprunt, One Nanosecond Photon Correlation Spectroscopy on Smectic Liquid Crystal Films, Phys. Rev. Lett. 94, 067801 (2005).

[53] C. Roland, R. Bogoslovov, R. Casalini, A. Ellis, S. Bair, S. Rzoska, K. Czuprynski, and S. Urban, Thermodynamic scaling and the characteristic relaxation time at the phase transition of liquid crystals, J. Chem. Phys. 128, 224506 (2008).

[54] V. Rühle, C. Junghans, A. Lukyanov, K. Kremer, and D. Andrienko, Versatile object-oriented toolkit for coarse-graining applications, J. Chem. Theory Comput. 5, 3211 (2009).

[55] M. Sakamoto and I. Hanasaki, Buckling instability of smectic lamellar phase affected by the constituent molecular shape, Jpn. J. Appl. Phys. 58, SDDC03 (2019).

[56] S. Miyajima, K. Nakazawa, K. Niikura, Y. Ujiiye, M. Yashiro, and T. Chiba, Effect of terminal polar substituents on the nature of smectic a liquid crystals of a series of 4-(4"octyloxybenzoyloxy) benzylidene-4'-substituted anilines, Liq. Cryst. 8, 707 (1990).

[57] E. Brochu, V. M. Cora, and N. De Freitas, A tutorial on bayesian optimization of expensive cost functions, with application to active user modeling and hierarchical reinforcement learning, arXiv:1012.2599.

[58] M. R. Wilson, Molecular dynamics simulations of flexible liquid crystal molecules using a gay-berne/lennard-jones model, J. Chem. Phys. 107, 8654 (1997).

[59] D. Ruppel and E. Sackmann, On defects in different phases of two-dimensional lipid bilayers, J. Phys. 44, 1025 (1983).

[60] C. Harrison, Z. Cheng, S. Sethuraman, D. A. Huse, P. M. Chaikin, D. A. Vega, J. M. Sebastian, R. A. Register, and D. H. Adamson, Dynamics of pattern coarsening in a two-dimensional smectic system, Phys. Rev. E 66, 011706 (2002).

[61] P. G. de Gennes and J. Prost, The Physics of Liquid Crystals (Clarendon Press, Oxford, 1993). 